\title{
DARBOUX EVALUATIONS OF ALGEBRAIC GAUSS HYPERGEOMETRIC FUNCTIONS
}

\author{
Raimundas VIDŪNAS
}

(Received 25 May 2012)

\begin{abstract}
Algebraic Gauss hypergeometric functions can be expressed explicitly in several ways. One attractive way is to pull-back their hypergeometric equations (with a finite monodromy) to Fuchsian equations with a finite cyclic monodromy, and express the algebraic solutions as radical functions on the covering curve. This article presents these pull-back transformations of minimal degree for the hypergeometric equations with the tetrahedral, octahedral or icosahedral projective monodromy. The minimal degree is 4,6 or 12, respectively. The covering curves are called Darboux curves, and they have genus zero or (for some icosahedral Schwarz types) genus one.
\end{abstract}

\section{Introduction}

Algebraic Gauss hypergeometric functions were studied by many authors [BD79, Bou98, Bri77, Fuc75, Kat72, Kle78, Pep81, Sch72, SU93, vdPU98]. The purpose of this article is to derive and present satisfying explicit forms for these functions.

The Gauss hypergeometric equation is

$$
X(1-X) \frac{d^{2} Y(X)}{d X^{2}}+(c-(a+b+1) Z) \frac{d Y(X)}{d X}-a b Y(X)=0 .
$$

This is a Fuchsian equation on $\mathbb{P}_{X}^{1}$ with three regular singular points $X=0,1, \infty$. The local exponent differences are: $1-c$ at $X=0 ; c-a-b$ at $X=1$; and $a-b$ at $X=\infty$. Let $E\left(e_{1}, e_{2}, e_{3}\right)$ denote a hypergeometric equation with the local exponent differences $e_{1}, e_{2}, e_{3}$ at the three singular points. The order and multiplication by -1 of the exponent differences is disregarded in this notation.

As is well known [BD79, Sch72, vdPU98], hypergeometric equation (1.1) has a basis of algebraic solutions if and only if its monodromy group is finite. The following hypergeometric equations (and their fractional-linear transformations; see Appendix A.4) have this property and are called standard hypergeometric equations with algebraic solutions:

- $E(1,1 / n, 1 / n)$, where $n$ is a positive integer; the hypergeometric equation degenerates to a Fuchsian equation with two singular points; its monodromy group is a cyclic group with $n$ elements;

2010 Mathematics Subject Classification: Primary 33C05, 34A30.

Keywords: Gauss hypergeometric function; Schwarz list; monodromy; pull-back transformations.

(C) 2013 Faculty of Mathematics, Kyushu University 
- $E(1 / 2,1 / 2,1 / n)$, where $n$ is an integer, $n \geq 2$; the projective monodromy group of this equation is the dihedral group with $2 n$ elements;

- $E(1 / 2,1 / 3,1 / 3)$; the projective monodromy is the tetrahedral group, isomorphic to $A_{4}$;

- $E(1 / 2,1 / 3,1 / 4)$; the projective monodromy is the octahedral group, isomorphic to $S_{4}$;

- $E(1 / 2,1 / 3,1 / 5)$; the projective monodromy is the icosahedral group, isomorphic to $A_{5}$.

Recall that the projective monodromy group is the monodromy group (or the Galois group, if solutions are algebraic) of $y_{1} / y_{2}$, where $y_{1}, y_{2}$ is a basis of solutions of the differential equation. The quotient $y_{1} / y_{2}$ is known as a Schwarz map. In general, the two-dimensional monodromy representation of a second-order Fuchsian equation is a cyclic extension of the projective monodromy.

The celebrated theorem of Klein [Kle77] states that if a second-order linear homogeneous differential equation only has algebraic solutions, then that equation is a pullback transformation of a standard hypergeometric equation from the list above. Explicitly, if the Fuchsian equation has coefficients in $\mathbb{C}(X)$, the pull-back transformation changes the variable $Z$ in (1.1) to a rational function $\varphi(X)$. In geometric terms, we have a finite covering $\varphi: \mathbb{P}_{X}^{1} \rightarrow \mathbb{P}_{Z}^{1}$ between two projective lines, and we pull-back the standard hypergeometric equation from $\mathbb{P}_{Z}^{1}$ onto $\mathbb{P}_{X}^{1}$.

The possible finite projective monodromies for the second-order Fuchsian equations are the same as listed above: a cyclic, a dihedral, or the tetrahedral, octahedral or icosahedral groups. The standard hypergeometric equation in Klein's theorem can be chosen to have the same projective monodromy. In particular, the theorem of Klein implies that if a hypergeometric equation only has algebraic solutions, then it is a pull-back transformation of a listed standard hypergeometric equation with the same projective monodromy.

Hypergeometric equations with finite monodromies were first classified by Schwarz [Sch72]. Disregarding hypergeometric equations with a cyclic monodromy, Schwarz gave a list of 15 types of these hypergeometric equations. One type consists of hypergeometric equations with a dihedral projective monodromy. The other types are represented by the following hypergeometric equations:

- $E(1 / 2,1 / 3,1 / 3), E(1 / 3,1 / 3,2 / 3)$; the projective monodromy is tetrahedral;

- $E(1 / 2,1 / 3,1 / 4), E(2 / 3,1 / 4,1 / 4)$; the projective monodromy is octahedral;

- $E(1 / 2,1 / 3,1 / 5), E(1 / 2,1 / 3,2 / 5), E(1 / 2,1 / 5,2 / 5), E(1 / 3,1 / 3,2 / 5)$, $E(1 / 3,2 / 3,1 / 5), E(2 / 3,1 / 5,1 / 5), E(1 / 3,2 / 5,3 / 5), E(1 / 3,1 / 5,3 / 5)$, $E(1 / 5,1 / 5,4 / 5), E(2 / 5,2 / 5,2 / 5)$; the projective monodromy is icosahedral.

We refer to a Schwarz type of hypergeometric equations with algebraic solutions by the triple of the exponent differences $e_{1}, e_{2}, e_{3}$ of these representative equations. (Usually, the Schwarz type is denoted by a roman numeral from I to XV.) We refer to the listed 14 hypergeometric equations as main representatives of the Schwarz types. Hypergeometric equations of the 
same Schwarz type are characterized by the property that their hypergeometric solutions are contiguous to hypergeometric solutions of the main representative (see Appendix A.6).

Algebraic solutions of differential equations can be represented in several ways, for example by minimal polynomial equations [SU93] or (if the Galois group is solvable) by nested radical expressions. In [BvHW03], [vHW05] and [Ber04, Ch. 1] algorithms are developed to represent algebraic solutions of second-order linear differential equations using Klein's theorem. The representation form is

$$
\theta(x) H(\varphi(x)),
$$

where $\varphi(x)$ is a rational function, $\theta(x)$ is a radical function that define Klein's pull-back transformation, and $H(z)$ is a solution of a corresponding standard hypergeometric equation.

We propose to pull-back a hypergeometric equation with a finite monodromy to Fuchsian equations with a cyclic monodromy group. Then some hypergeometric solutions are transformed to rather simple radical functions. We call a pull-back covering $\phi: D \rightarrow \mathbb{P}^{1}$ of this kind a Darboux covering. The covering curve $D$ is called a Darboux curve. Identification of algebraic Gauss hypergeometric functions with radical functions on an algebraic curve offers satisfying geometric intuition, especially when the Darboux covering has low degree. We use the term Darboux evaluation to refer to the aspired identification of hypergeometric functions with radical functions.

The Darboux coverings for hypergeometric equations with finite dihedral projective monodromies have degree two. They are simply $Z=x^{2}$ if half-integer exponent differences are at $Z=0$ and $Z=\infty$. In [Vid11], the pulled-back dihedral hypergeometric functions are expressed in terms of power functions and terminating Appell's (double hypergeometric) $F_{2}$ or $F_{3}$ sums.

Theory of Darboux coverings is developed in Section 3. It turns out that Darboux coverings for hypergeometric equations of the same Schwarz type are identical. Therefore, we have finitely many different Darboux coverings and Darboux curves. We compute all Darboux coverings of minimal degree, which turns out to be 4,6 or 12 for the tetrahedral, octahedral and icosahedral types, respectively. The corresponding Darboux curves have genus zero or (for some icosahedral types) genus one. For each Schwarz type, we use Darboux coverings of minimal degree and compute Darboux evaluations for two hypergeometric solutions of the main representative equation. The evaluations are presented in Section 2. Using these formulas, differentiation and contiguous relations, one can compute Darboux evaluations for two different hypergeometric solutions of any hypergeometric equation with the tetrahedral, octahedral or icosahedral projective monodromy.

Section 4 presents the method used to compute Darboux evaluations. Most attention is paid to describing complicated computations on genus 1 Darboux curves. The computer algebra system Maple was used in the computations. Appendix A gives short introductions to pull-back transformations, Fuchsian equations, contiguous relations and other relevant topics.

\section{Hypergeometric formulas}

This section presents the main explicit results: Darboux evaluations for two hypergeometric functions of each Schwarz type with the tetrahedral, octahedral and icosahedral projective 
monodromies. Each pair of hypergeometric evaluations forms a solution basis of the main representative equation of the respective Schwarz type, up to a power factor to the second evaluation. Darboux evaluations for other hypergeometric functions of the same Schwarz type can be computed by using contiguous relations if another evaluation in the same contiguous orbit is known. Another contiguous evaluation can be computed by differentiation.

We use Darboux coverings of minimal possible degree, which is 4, 6 and 12 for the 3 considered monodromies, respectively, as shown in Lemma 3.3. For the tetrahedral and octahedral Schwarz types, Darboux coverings are evident from the arguments of the hypergeometric functions. Darboux coverings for icosahedral Schwarz types are given in (2.9), (2.14), (2.18), (2.24), (2.30), and (2.34). Some of these Darboux coverings are valid for two different Schwarz types. For seven icosahedral types, the Darboux curve has genus one rather than zero. Weierstrass equations $\xi^{2}=x\left(1+\alpha x+\beta x^{2}\right)$ with $\alpha, \beta \in \mathbb{C}$ for these curves are given in (2.17), (2.23), (2.29), and (2.33). The Darboux evaluations hold locally around $x=0$ or, if the Darboux curve has genus one, around the point $(x, \xi)=(0,0)$. A simple way to check each evaluation is to expand both sides in power series around $x=0$. If the Darboux curve has genus one, one has to replace $\xi$ by the respective $\sqrt{x} \sqrt{1+\alpha x+\beta x^{2}}$, and expand the power series in $\sqrt{x}$.

\subsection{Tetrahedral hypergeometric equations}

Here are two hypergeometric evaluations for solutions of $E(1 / 2,1 / 3,1 / 3)$ :

$$
\begin{aligned}
{ }_{2} \mathrm{~F}_{1}\left(\begin{array}{c|c}
1 / 4,-1 / 12 & \frac{x(x+4)^{3}}{4(2 x-1)^{3}}
\end{array}\right) & =(1-2 x)^{-1 / 4}, \\
{ }_{2} \mathrm{~F}_{1}\left(\begin{array}{c}
1 / 4,7 / 12 \\
4 / 3
\end{array}\right. & \left.\frac{x(x+4)^{3}}{4(2 x-1)^{3}}\right)=\frac{1}{1+x / 4}(1-2 x)^{3 / 4} .
\end{aligned}
$$

If we multiply the second formula by $x^{1 / 3}(x+4) /(2 x-1)$, the two evaluated functions form a basis of solutions of the same hypergeometric equation. Here are two hypergeometric evaluations for solutions of $E(1 / 3,1 / 3,2 / 3)$, a representative of the other tetrahedral Schwarz type:

$$
\begin{aligned}
& { }_{2} \mathrm{~F}_{1}\left(\begin{array}{c|c}
1 / 2,-1 / 6 & \frac{x(x+2)^{3}}{2 / 3}
\end{array}\right)=(1+2 x)^{-1 / 2}, \\
& { }_{2} \mathrm{~F}_{1}\left(\begin{array}{c|c}
1 / 6,5 / 6 & x(x+2)^{3} \\
4 / 3 & \frac{1}{(2 x+1)^{3}}
\end{array}\right)=\frac{1}{1+x / 2}(1+2 x)^{1 / 2}(1+x)^{1 / 3} .
\end{aligned}
$$

\subsection{Octahedral hypergeometric equations}

Here are two representative solutions of $E(1 / 2,1 / 3,1 / 4)$ :

$$
\begin{aligned}
& { }_{2} \mathrm{~F}_{1}\left(\begin{array}{c|c}
7 / 24,-1 / 24 \\
3 / 4
\end{array} \mid \frac{108 x(x-1)^{4}}{\left(x^{2}+14 x+1\right)^{3}}\right)=\left(1+14 x+x^{2}\right)^{-1 / 8}, \\
& { }_{2} \mathrm{~F}_{1}\left(\begin{array}{c|c}
5 / 24,13 / 24 \\
5 / 4
\end{array} \mid \frac{108 x(x-1)^{4}}{\left(x^{2}+14 x+1\right)^{3}}\right)=\frac{1}{1-x}\left(1+14 x+x^{2}\right)^{5 / 8} .
\end{aligned}
$$


And here are two representative solutions of $E(1 / 4,1 / 4,2 / 3)$ :

$$
\begin{aligned}
& { }_{2} \mathrm{~F}_{1}\left(\begin{array}{c|c}
7 / 12,-1 / 12 & \frac{27 x(x+1)^{4}}{3 / 4} \\
2\left(x^{2}+4 x+1\right)^{3}
\end{array}\right)=\frac{(1+x / 2)^{1 / 4}}{\left(1+4 x+x^{2}\right)^{1 / 4}},
\end{aligned}
$$

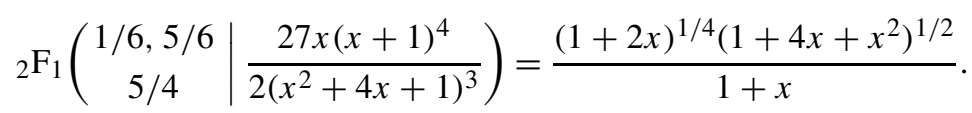

\subsection{Icosahedral hypergeometric equations}

The Darboux covering for hypergeometric equations of the Schwarz types $(1 / 2,1 / 3,1 / 5)$ and $(1 / 2,1 / 3,2 / 5)$ is

$$
\varphi_{1}(x)=\frac{1728 x\left(x^{2}-11 x-1\right)^{5}}{\left(x^{4}+228 x^{3}+494 x^{2}-228 x+1\right)^{3}}
$$

Representative evaluations for solutions of $E(1 / 2,1 / 3,1 / 5)$ are

$$
\begin{gathered}
{ }_{2} \mathrm{~F}_{1}\left(\begin{array}{c|c}
19 / 60,-1 / 60 \\
4 / 5
\end{array} \mid \varphi_{1}(x)\right)=\left(1-228 x+494 x^{2}+228 x^{3}+x^{4}\right)^{-1 / 20}, \\
{ }_{2} \mathrm{~F}_{1}\left(\begin{array}{c}
11 / 60,31 / 60 \\
6 / 5
\end{array} \mid \varphi_{1}(x)\right)=\frac{\left(1-228 x+494 x^{2}+228 x^{3}+x^{4}\right)^{11 / 20}}{1+11 x-x^{2}} .
\end{gathered}
$$

Similarly, basic evaluations for solutions of $E(1 / 2,1 / 3,2 / 5)$ are

$$
\begin{aligned}
{ }_{2} \mathrm{~F}_{1}\left(\begin{array}{c}
13 / 60,-7 / 60 \\
3 / 5
\end{array} \mid \varphi_{1}(x)\right) & =\frac{1-7 x}{\left(1-228 x+494 x^{2}+228 x^{3}+x^{4}\right)^{7 / 20}}, \\
{ }_{2} \mathrm{~F}_{1}\left(\begin{array}{c}
17 / 60,37 / 60 \\
7 / 5
\end{array} \mid \varphi_{1}(x)\right) & =\frac{\left(1+\frac{1}{7} x\right)\left(1-228 x+494 x^{2}+228 x^{3}+x^{4}\right)^{17 / 20}}{\left(1+11 x-x^{2}\right)^{2}} .
\end{aligned}
$$

The Darboux covering for Schwarz type $(1 / 2,1 / 5,2 / 5)$ is the following:

$$
\varphi_{2}(x)=\frac{64 x\left(x^{2}-x-1\right)^{5}}{\left(x^{2}-1\right)\left(x^{2}+4 x-1\right)^{5}} .
$$

Representative evaluations for solutions of $E(1 / 2,1 / 5,2 / 5)$ are

$$
\begin{aligned}
& { }_{2} \mathrm{~F}_{1}\left(\begin{array}{c}
7 / 20,-1 / 20 \\
4 / 5
\end{array} \mid \varphi_{2}(x)\right)=\frac{(1+x)^{7 / 20}}{(1-x)^{1 / 20}\left(1-4 x-x^{2}\right)^{1 / 4}}, \\
& { }_{2} \mathrm{~F}_{1}\left(\begin{array}{c}
3 / 20,11 / 20 \\
6 / 5
\end{array} \mid \varphi_{2}(x)\right)=\frac{(1+x)^{3 / 20}(1-x)^{11 / 20}\left(1-4 x-x^{2}\right)^{3 / 4}}{1+x-x^{2}} .
\end{aligned}
$$

Darboux curves for the other icosahedral Schwarz types have genus one. The Darboux curve for hypergeometric equations of the Schwarz types $(1 / 3,1 / 3,2 / 5)$ and (1/3, 2/3, 1/5) is given by the equation

$$
C_{3}: \quad \xi^{2}=x\left(1+33 x-9 x^{2}\right) .
$$

The Darboux covering is

$$
\varphi_{3}(x, \xi)=\frac{144 \xi\left(1+33 x-9 x^{2}\right)^{2}(1-9 \xi+54 x)}{\left(1+21 \xi-117 x+9 x \xi-234 x^{2}\right)^{3}}
$$


Here are basic evaluations for solutions of $E(1 / 3,1 / 3,2 / 5)$ :

$$
\begin{aligned}
{ }_{2} \mathrm{~F}_{1}\left(\begin{array}{c}
3 / 10,-1 / 30 \\
3 / 5
\end{array} \mid \varphi_{3}(x, \xi)\right) & =\frac{(1-9 \xi+54 x)^{1 / 30}}{\left(1+21 \xi-117 x+9 x \xi-234 x^{2}\right)^{1 / 10}}, \\
{ }_{2} \mathrm{~F}_{1}\left(\begin{array}{c}
7 / 10,11 / 30 \\
7 / 5
\end{array} \mid \varphi_{3}(x, \xi)\right) & =\frac{\left(1+21 \xi-117 x+9 x \xi-234 x^{2}\right)^{11 / 10}}{(1-9 \xi+54 x)^{11 / 30}\left(1+33 x-9 x^{2}\right)} .
\end{aligned}
$$

And here are basic evaluations for solutions of $E(1 / 3,2 / 3,1 / 5)$ :

$$
\begin{aligned}
& { }_{2} \mathrm{~F}_{1}\left(\begin{array}{c}
17 / 30,-1 / 10 \\
4 / 5
\end{array} \mid \varphi_{3}(x, \xi)\right)=\frac{(1-9 \xi+54 x)^{13 / 30}}{\left(1+21 \xi-117 x+9 x \xi-234 x^{2}\right)^{3 / 10}}, \\
& { }_{2} \mathrm{~F}_{1}\left(\begin{array}{c}
1 / 10,23 / 30 \\
6 / 5
\end{array} \mid \varphi_{3}(x, \xi)\right) \\
& \quad=\frac{\left(1+21 \xi-117 x+9 x \xi-234 x^{2}\right)^{3 / 10}(1-9 \xi+54 x)^{7 / 30}(\xi+5 x)}{\xi(1+9 x)} .
\end{aligned}
$$

The Darboux curve for the Schwarz types $(2 / 3,1 / 5,1 / 5)$ and $(1 / 3,2 / 5,3 / 5)$ is given by

$$
C_{4}: \quad \xi^{2}=x\left(1+5 x-5 x^{2}\right)
$$

The Darboux covering is

$$
\varphi_{4}(x, \xi)=\frac{432 x\left(1-\frac{7}{5} \xi-9 x-x^{2}\right)^{5}\left(1+50 x-125 \xi^{2}+450 x \xi-500 x^{2}\right)}{(5 \xi+57 x)\left(1+\frac{18}{5} \xi-16 x+x^{2}\right)^{5}\left(1+50 x-125 \xi^{2}-450 x \xi-500 x^{2}\right)} .
$$

Basic evaluations for solutions of $E(2 / 3,1 / 5,1 / 5)$ are

$$
\begin{gathered}
{ }_{2} \mathrm{~F}_{1}\left(\begin{array}{c|c}
1 / 6,-1 / 30 \\
4 / 5
\end{array} \mid \varphi_{4}(x, \xi)\right) \\
=\frac{\left(1-\frac{3}{5} \xi-\frac{34}{5} x\right)^{1 / 6}}{(1+3 \xi-20 x)^{1 / 6}\left(1+50 x-125 \xi^{2}-450 x \xi-500 x^{2}\right)^{1 / 30}}, \\
{ }_{2} \mathrm{~F}_{1}\left(\begin{array}{c}
1 / 6,11 / 30 \\
6 / 5
\end{array} \mid \varphi_{4}(x, \xi)\right)=\left(1+50 x-125 \xi^{2}-450 x \xi-500 x^{2}\right)^{11 / 30} \\
\times \frac{(1+3 \xi-20 x)^{5 / 6}\left(1-\frac{3}{5} \xi-\frac{34}{5} x\right)^{1 / 6}\left(1+\frac{21}{4} \xi+\frac{41}{4} x\right)}{(1-9 x)\left(1-\frac{7}{4} \xi-\frac{15}{2} x\right)(1+5 \xi+10 x)} .
\end{gathered}
$$

Basic evaluations for solutions of $E(1 / 3,2 / 5,3 / 5)$ are

$$
\begin{gathered}
{ }_{2} \mathrm{~F}_{1}\left(\begin{array}{c}
13 / 30,-1 / 6 \\
3 / 5
\end{array} \mid \varphi_{4}(x, \xi)\right) \\
=\frac{\left(1+50 x-125 \xi^{2}-450 x \xi-500 x^{2}\right)^{13 / 30}(1-3 \xi+2 x)}{(1+3 \xi-20 x)^{5 / 6}\left(1-\frac{3}{5} \xi-\frac{34}{5} x\right)^{1 / 6}(1+5 \xi+10 x)}, \\
{ }_{2} \mathrm{~F}_{1}\left(\begin{array}{c}
5 / 6,7 / 30 \\
7 / 5
\end{array} \mid \varphi_{4}(x, \xi)\right)=\left(1+50 x-125 \xi^{2}-450 x \xi-500 x^{2}\right)^{7 / 30} \\
\times \frac{\left(1+\frac{18}{5} \xi-16 x+x^{2}\right)^{7 / 6}\left(1+\frac{1}{25} x\right)^{5 / 6}(1+5 \xi+10 x)}{\left(1-\frac{7}{5} \xi-9 x-x^{2}\right)^{2}(1-5 x)^{7 / 6}} .
\end{gathered}
$$


The Darboux curve for the Schwarz type $(1 / 3,1 / 5,3 / 5)$ is given by

$$
C_{5}: \quad \xi^{2}=x(1+x)(1+16 x) .
$$

The Darboux covering is

$$
\varphi_{5}(x, \xi)=-\frac{54(\xi+5 x)^{3}(1-2 \xi+6 x)^{5}}{\left(1-16 x^{2}\right)(\xi-5 x)^{2}(1-2 \xi-14 x)^{5}} .
$$

Representative evaluations for solutions of $E(1 / 3,1 / 5,3 / 5)$ are

$$
\begin{aligned}
{ }_{2} \mathrm{~F}_{1}\left(\begin{array}{c}
8 / 15,-1 / 15 \\
4 / 5
\end{array} \mid \varphi 5(x, \xi)\right) & =\frac{(1+4 x)^{8 / 15}(\xi+5 x)^{1 / 6} x^{1 / 15}}{(1-2 \xi-14 x)^{1 / 3}(\xi-3 x)^{3 / 10}}, \\
{ }_{2} \mathrm{~F}_{1}\left(\begin{array}{c}
2 / 15,11 / 15 \\
6 / 5
\end{array} \mid \varphi_{5}(x, \xi)\right) & =\frac{(1-\xi+x)(1-2 \xi-14 x)^{2 / 3}(\xi+5 x)^{1 / 6}(\xi-3 x)^{13 / 10}}{(1+\xi+x)(1-2 \xi+6 x)(1+4 x)^{13 / 15} x^{11 / 15}} .
\end{aligned}
$$

The Darboux curve for the Schwarz types $(1 / 5,1 / 5,4 / 5)$ and $(2 / 5,2 / 5,2 / 5)$ is given by

$$
C_{6}: \quad \xi^{2}=x\left(1+x-x^{2}\right)
$$

The Darboux covering is

$$
\varphi_{6}(x, \xi)=\frac{16 \xi\left(1+x-x^{2}\right)^{2}(1-\xi)^{2}}{(1+\xi+2 x)(1+\xi-2 x)^{5}} .
$$

Basic evaluations for solutions of $E(1 / 5,1 / 5,4 / 5)$ are

$$
\begin{aligned}
{ }_{2} \mathrm{~F}_{1}\left(\begin{array}{c}
7 / 10,-1 / 10 \\
4 / 5
\end{array} \mid \varphi_{6}(x, \xi)\right) & =\frac{(1-\xi+2 x)^{1 / 15}(1-\xi)^{3 / 5}}{(1+\xi+2 x)^{7 / 30} \sqrt{1+\xi-2 x}} \\
{ }_{2} \mathrm{~F}_{1}\left(\begin{array}{c}
1 / 10,9 / 10 \\
6 / 5
\end{array} \mid \varphi_{6}(x, \xi)\right) & =\frac{\left(\xi+2 x+x^{2}\right)(1+\xi)^{1 / 10}(1-\xi)^{3 / 10}}{\xi(1-\xi+2 x)^{1 / 30}(1+\xi+2 x)^{2 / 15} \sqrt{1+\xi-2 x}}
\end{aligned}
$$

Basic evaluations for solutions of $E(2 / 5,2 / 5,2 / 5)$ are

$$
\begin{aligned}
{ }_{2} \mathrm{~F}_{1}\left(\begin{array}{c|c}
3 / 10,-1 / 10 \\
3 / 5
\end{array} \mid \varphi_{6}(x, \xi)\right) & =\frac{(1-\xi+2 x)^{2 / 15}(1+\xi+2 x)^{1 / 30}(1-\xi)^{1 / 5}}{\sqrt{1+\xi-2 x}} \\
{ }_{2} \mathrm{~F}_{1}\left(\begin{array}{c}
3 / 10,7 / 10 \\
7 / 5
\end{array} \mid \varphi_{6}(x, \xi)\right) & =\frac{(1+\xi+2 x)^{7 / 30}(1+\xi)^{1 / 5}(1+\xi-2 x)^{3 / 2}}{(1-\xi+2 x)^{1 / 15}(1-\xi)^{2 / 5}\left(1+x-x^{2}\right)}
\end{aligned}
$$

\section{Darboux curves}

In the context of integration theory of vector fields [Dar78, Oll01], one considers a polynomial vector field on $\mathbb{C}^{2}$ given by a derivation $\mathcal{L}=f(x, y) \partial / \partial x+g(x, y) \partial / \partial y$ with $f, g \in \mathbb{C}[x, y]$. A polynomial $p(x, y)$ is called a Darboux polynomial for the vector field (or for the derivation $\mathcal{L})$ if $p(x, y)$ divides $\mathcal{L} p(x, y)$ in the ring $\mathbb{C}[x, y]$. An algebraic curve defined as the zero set of a Darboux polynomial is called a Darboux curve. A Darboux curve is infinitesimally invariant under the vector field. Hence, an alternative term is invariant algebraic curve, as in [CLPZ02] for example. 
In differential Galois theory we have the following definition [Sin92, Wei95]. Let $K$ be a differential field, and let $R=K\left[y_{1}, \ldots, y_{n}\right]$ be a differential ring. Let $\mathcal{D}$ denote the derivation on $R$, and suppose that it extends the derivation on $K$. Then $p \in R$ is a Darboux polynomial for $\mathcal{D}$ if $p$ divides $\mathcal{D} p$ in $R$. For example, consider the differential equation

$$
y^{(n)}+a_{n-1} y^{(n-1)}+\cdots+a_{1} y^{\prime}+a_{0} y=0, \quad \text { with } a_{0}, a_{1}, \ldots, a_{n-1} \in K .
$$

Let $\mathcal{D}$ be the derivation on $K\left[y, y^{\prime}, \ldots, y^{(n-1)}\right]$ defined by $\mathcal{D} y=y^{\prime}, \quad \mathcal{D} y^{\prime}=$ $y^{\prime \prime}, \ldots, \mathcal{D} y^{(n-2)}=y^{(n-1)}, \mathcal{D} y^{(n-1)}=-a_{n-1} y^{(n-1)}-\cdots-a_{1} y^{\prime}-a_{0} y$. Darboux polynomials for this derivation correspond to semi-invariants of the differential Galois group for (3.1); see [Wei95, Theorem 38]. If the order $n$ of (3.1) is equal to 2, then one considers Darboux polynomials in $K[u]$ for the derivation $\mathcal{D}$ defined by $\mathcal{D} u=-u^{2}-a_{1} u-a_{0}$. In [UW96, Wei94] these Darboux polynomials are called special polynomials.

As we see, the terms 'Darboux polynomials' and 'Darboux curves' are not consistently used although there is a common theme. In the context of algebraic solutions of differential equations, we wish to use the term 'Darboux curves' to offer a geometric guidance. Hence, the following definitions.

Definition 3.1. Let $C$ denote an algebraic curve (see Appendix A.1). We suppose that the function field $\mathbb{C}(C)$ is a differential field. Consider differential equation (3.1) on $\mathbb{P}^{1}$, assuming $K=\mathbb{C}\left(\mathbb{P}^{1}\right)$. We say that a finite covering $\phi: C \rightarrow \mathbb{P}^{1}$ is a Darboux covering for (3.1), if a pull-back of (3.1) with respect to $\phi$ has a solution $Y$ such that:

(i) its logarithmic derivative $u=Y^{\prime} / Y$ is in $\mathbb{C}(C)$;

(ii) the algebraic degree of $u$ over $K$ is precisely the degree of $\phi$.

In the described situation, $C$ is called a Darboux curve for (3.1).

To see the connection with previous definitions, let us assume that the order $n$ of (3.1) is equal to 2 . We take the differential field $K=\mathbb{C}(z)$ with $z^{\prime}=1$. Suppose that we have a Darboux covering $\phi: C \rightarrow \mathbb{P}^{1}$ of degree $d$, and let $u$ be a required logarithmic derivative in $\mathbb{C}(C)$. Then we have the following facts.

- The logarithmic derivative $u$ is an algebraic solution of the associated Riccati equation $u^{\prime}+u^{2}+a_{1} u+a_{0}=0$. Let $P(u)=0$ denote the minimal monic polynomial equation defining $u$ over $K$. The polynomial $P$ has degree $d$, and it is a defining equation for the Darboux curve $C$. Therefore, $\mathbb{C}(C)=\mathbb{C}(z, u)$.

- The polynomial $P$ is a Darboux polynomial for the derivation $\mathcal{D} u=-u^{2}-a_{1} u-a_{0}$ in the definition of differential Galois theory; see [UW96, Lemma 2.4].

- The expression $y^{n+1} P\left(y^{\prime} / y\right)$ is a homogeneous polynomial in $K\left[y, y^{\prime}\right]$. It is a Darboux polynomial for the corresponding derivation on $K\left[y, y^{\prime}\right]$.

- Assume that $a_{1}, a_{0} \in \mathbb{C}[z]$, and consider the vector field $\partial / \partial z-\left(u^{2}+a_{1} u+a_{0}\right) \partial / \partial u$. Then $P$ is a Darboux polynomial according the first definition above.

In addition, we have the following facts.

- Suppose that $P=u^{m}+\sum_{j=0}^{d-1} b_{j} u^{j}$. Then $-b_{d-1}$ is the logarithmic derivative of an exponential solution of the $d$ th symmetric tensor power of differential equation (3.1); see [UW96, Theorem 2.1] or [SU93, Section 3.2]. All other coefficients $b_{j}$ are determined by $b_{d-1}$ and the differential equation [UW96, Section 2]. 
- The mentioned exponential solution (which can be expressed as $\exp \int-b_{d-1}$ ) is a degree $d$ semi-invariant of the differential Galois group [UW96, Section 2].

- Inside a Picard-Vessiot extension for (3.1), the field $\mathbb{C}(C)$ is fixed by a 1-reducible subgroup of the differential Galois group for (3.1) of finite index [SU93, Lemma 3.1].

- If the differential Galois group of (3.1) is finite, the 1-reducible subgroups are cyclic subgroups [UW96, Lemma 1.5].

As we see, Darboux coverings correspond to algebraic Riccati solutions and semi-invariants of the differential Galois group.

We defined Darboux curves and coverings for general linear differential equations. They can be computed from minimal polynomials for Riccati solutions or by direct solution of pulled-back equations. Pulled-back equations on Darboux curves have cyclic monodromy groups, hence their solutions can be expressed in terms of radical functions. Solutions of the original equation can be presented as those radical solutions of the transformed equation, keeping in mind the inverse substitution defined by the Darboux covering. We refer to these radical expressions as Darboux evaluations of solutions of the original equation. They could be satisfying for many purposes. Darboux curves of genus zero can be parameterized, and Weierstrass forms of genus one Darboux used. Thanks to contiguous relations, Darboux evaluations for algebraic hypergeometric functions can be derived using only a few representative Darboux evaluations for each Schwarz type.

Darboux evaluations for solutions of hypergeometric equations with dihedral projective monodromies is recognized in [Vid11] or [Erd53, 2.5.5]. The Darboux coverings in the dihedral case are simple quadratic coverings, such as $\varphi(x)=x^{2}$. The Darboux curves and coverings for hypergeometric equations were introduced earlier in [Vid99, Section 4.2]. The Darboux coverings of minimal degree for all tetrahedral, octahedral types and for the icosahedral types $(1 / 2,1 / 3,1 / 5),(1 / 2,1 / 3,2 / 5),(1 / 2,1 / 5,2 / 5),(1 / 3,1 / 3,2 / 5)$, and $(1 / 3,1 / 3,2 / 5)$ were computed in that $\mathrm{PhD}$ thesis.

\subsection{Basic properties of Darboux curves}

The following lemmas identify Darboux coverings for hypergeometric equations of the same Schwarz type, and imply that there are only finitely many different Darboux coverings for all hypergeometric equations. These are key attractive features for using Darboux pulls-backs.

LEMMA 3.2. Suppose that $\phi: D \rightarrow \mathbb{P}^{1}$ is a Darboux covering for a hypergeometric equation $E_{1}$ with a tetrahedral, octahedral or icosahedral monodromy, and $E_{2}$ is hypergeometric equation of the same Schwarz type. Then $\phi: D \rightarrow \mathbb{P}^{1}$ is a Darboux covering for $E_{2}$ as well.

Proof. Let $y_{1}$ be a hypergeometric solution of $E_{1}$. Since $E_{1}$ and $E_{2}$ have the same Schwarz type, there is a hypergeometric solution $y_{2}$ which is contiguous to $y_{1}$. Recall that $y_{1}^{\prime}$ is contiguous to $y_{1}$ as well. Therefore, there is a contiguous relation $y_{2}=a y_{1}+b y_{1}^{\prime}$, with $a, b \in \mathbb{C}(z)$. There is also a contiguity relation $y_{2}^{\prime}=c y_{1}+d y_{1}^{\prime}$, with $c, d \in \mathbb{C}(z)$. Let $u_{1}$ denote the Riccati solution $y_{1}^{\prime} / y_{1}$ for $E_{1}$, and let $u_{2}$ denote the Riccati solution $y_{2}^{\prime} / y_{2}$ for $E_{2}$. Then $u_{2}=\left(c+d u_{1}\right) /\left(a+b u_{1}\right)$. This implies that the function fields $\mathbb{C}\left(z, u_{1}\right)$ and $\mathbb{C}\left(z, u_{2}\right)$ are isomorphic. 
The following lemma categorizes all possible Darboux coverings for hypergeometric equations with the considered monodromies.

LEMMA 3.3. Suppose that the projective monodromy $G$ of a hypergeometric equation (1.1) is tetrahedral $A_{4}$, octahedral $S_{4}$ or icosahedral $A_{5}$.

- If $G \cong A_{4}$ (tetrahedral group), the Darboux coverings have degree 4, 6 or 12 .

- If $G \cong S_{4}$ (octahedral group), the Darboux coverings have degree 6, 8, 12 or 24 .

- If $G \cong A_{5}$ (icosahedral group), the Darboux coverings have degree 12, 20, 30 or 60 .

- The Darboux coverings of each degree are unique up to automorphisms of the Darboux curve.

Proof. As noted, Darboux curves correspond to algebraic Riccati solutions. According to [UW96, Corollary 1.7], the given numbers are possible degrees of minimal polynomials for solutions of the Riccati equation associated with the hypergeometric equations with considered monodromy groups. For each projective monodromy group, Riccati solutions of the maximal degree are not unique, but they define the same function field (because of irreducibility of the monodromy) and, hence, the same Darboux curve. Irreducible polynomials for Riccati solutions of other (non-maximal) degrees are unique except that there are two irreducible polynomials of degree four in the tetrahedral case. To show that those two degree-four polynomials define isomorphic Darboux curves, we first use Lemma 3.2 to reduce consideration to the two equations $E(1 / 2,1 / 3,1 / 3)$ and $E(1 / 3,1 / 3,2 / 3)$, representing the two distinct tetrahedral Schwarz types. The two points with the exponent differences $1 / 3$ of either of these equations are interchanged by a fractional-linear transformation (say, $X \mapsto 1-X)$, and the two minimal polynomials differ by the same transformation.

The uniqueness claim does not mean that coverings for pulls-backs of considered hypergeometric equations to Fuchsian equations with the isomorphic cyclic monodromies have to be isomorphic. In fact, Litcanu [Lit04, Section 2] observed that there are two pullback transformations of $E(1 / 2,1 / 3,1 / 4)$ to a Fuchsian equation with the $\mathbb{Z} / 2 \mathbb{Z}$ monodromy. One pull-back covering is the degree-12 Darboux covering

$$
\frac{27(x-1)^{4}\left(x^{2}+6 x+1\right)^{4}}{\left(x^{2}-10 x+1\right)^{3}\left(3 x^{2}+2 x+3\right)^{3}},
$$

and the other is $108 x^{2}\left(x^{2}-1\right)^{4} /\left(x^{4}+14 x^{2}+1\right)^{3}$. The latter covering is a composition of the degree-six covering for octahedral equations (for a pull-back to $\mathbb{Z} / 4 \mathbb{Z}$ ) and a quadratic covering. It is not a Darboux covering, because the respective Riccati solution is still of degree six. The two coverings of degree 12 have different branching patterns.

\subsection{Darboux coverings for standard hypergeometric equations}

Here are the main facts about Darboux coverings for standard hypergeometric equations.

LEMMA 3.4. Let H denote a standard hypergeometric equation with tetrahedral, octahedral or icosahedral differential Galois group G. Let $\varphi: D \rightarrow \mathbb{P}^{1}$ be a Darboux covering for $H$ of degree $m$. Then: 
(i) the Darboux curve D has genus zero;

(ii) let $\widetilde{\varphi}: \widetilde{D} \rightarrow \mathbb{P}^{1}$ be a Darboux covering for $H$ of the maximal degree $|G|$; then we have a factorization $\tilde{\varphi}=\gamma \circ \varphi$, where the covering $\gamma: \widetilde{D} \rightarrow D$ is given (up to fractionallinear transformations of $D$ and $\widetilde{D})$ by $x \mapsto x^{|G| / m}$;

(iii) let $X \in \mathbb{P}^{1}$ be a regular singular point of the hypergeometric equation; assume that the local exponent difference at $X$ has denominator $k$; then there are $\lfloor m / k\rfloor$ points above $X$ with branching index $k$, and other points above $X$ are unramified.

Proof. First we prove this theorem for the Darboux covering $\widetilde{\varphi}$ of maximal degree $m=|G|$. The first statement follows classically from [Kle84]. In particular, there is an action of $G$ on $\widetilde{D} \cong \mathbb{P}^{1}$, and the projection $\mathbb{P}^{1} \rightarrow \mathbb{P}^{1} / G \cong \mathbb{P}^{1}$ is precisely $\widetilde{\varphi}$. This map is also the inverse of a Schwarz map for the hypergeometric equation. At each fiber of $\widetilde{\varphi}$ the points have the same branching index, since $\mathbb{C}(\widetilde{D}) \supset \mathbb{C}[z]$ is a Galois extension. All exponent differences of a pull-back of $H$ with respect to $\widetilde{\varphi}$ are integers. A suitably normalized (by $\theta(x)$ ) pull-back of $H$ with respect to $\widetilde{\varphi}$ has trivial Galois group. As a consequence, in the situation of part (iii) the branching indices should be integer multiples of $k$. Since $g(\widetilde{D})=0$, Hurwitz theorem leaves only one possibility which is described in part (iii). Part (ii) is trivial in the considered case. The extension $\mathbb{C}(\widetilde{D}) \supset \mathbb{C}[z]$ is also a Picard-Vessiot extension for $H$.

Now we consider a general Darboux covering $\varphi: D \rightarrow \mathbb{P}^{1}$. Let $u$ be a corresponding Riccati solution of degree $m$, so that $\mathbb{C}(D) \cong \mathbb{C}(z, u)$. Let $K \supset \mathbb{C}(z)$ denote a PicardVessiot extension of $(1.1)$. We have $K \cong \mathbb{C}(\widetilde{D})$ as just above. Consider the tower of field extensions $K \supset \mathbb{C}(D) \supset \mathbb{C}(z)$. Here $K \supset \mathbb{C}(D)$ is a Picard-Vessiot extension for the differential equation $y^{\prime}=u y$, so its Galois group must be a cyclic subgroup of $G$ of index $m$. The existence of the corresponding covering $\gamma: \widetilde{D} \rightarrow D$ implies (i). Hurwitz theorem implies that there are exactly two branching points of $\gamma$. This implies (ii). We see that the equation $y^{\prime}=u y$ has exactly two singular points, so in the situation of part (iii) branching indices are equal to either $k$ or 1 , and that there in total only two unramified points above the three regular singular points of (1.1). This information gives part (iii).

The Darboux coverings for the standard hypergeometric equations can be computed from scratch, by using the branching pattern prescribed by part (iii) of Lemma 3.4, and Algorithm 1 in [Vid05, Section 3] for example. On the other hand, the coefficients in their rational expressions are familiar from the (semi)-invariants of the classical action of $A_{4}, S_{4}$, $A_{5}$ on $\mathbb{C}[x]$ (or on homogeneous polynomials in $\mathbb{C}[x, y]$ ); see [Kle84].

Explicit expressions for the Darboux coverings of the minimal degree 4, 6 or 12 are evident in formulas (2.1), (2.5), and (2.9), respectively. The covering of degree six for the standard tetrahedral equation is given by

$$
X \mapsto \frac{\left(x^{2}-6 x-3\right)^{3}}{\left(x^{2}+6 x-3\right)^{3}} .
$$

For the standard octahedral equation, the Darboux covering of degree eight is given by

$$
X \mapsto \frac{\left(x^{2}+20 x-8\right)^{4}}{256 x(x+1)^{3}(x-8)^{3}},
$$


while the covering of degree 12 is given in (3.2). The Darboux coverings of degree 20 and 30 for the standard icosahedral equation are given by the rational functions

$$
\begin{gathered}
\frac{64\left(x^{4}+55 x^{3}-165 x^{2}-275 x+25\right)^{5}}{125 x\left(x^{2}+5 x+40\right)^{3}\left(x^{2}-40 x-5\right)^{3}\left(8 x^{2}-5 x+5\right)^{3}}, \\
\frac{27\left(x^{2}+2 x+5\right)^{5}\left(x^{4}+20 x^{3}-210 x^{2}+100 x+25\right)^{5}}{\left(3 x^{2}-10 x+15\right)^{3}\left(x^{4}+70 x^{2}+25\right)^{3}\left(x^{4}-60 x^{3}-370 x^{2}-300 x+25\right)^{3}} .
\end{gathered}
$$

The Darboux coverings of the maximal degree $D \in\{12,24,60\}$ are obtained by composing an indicated Darboux covering of smaller degree $m$ with the cyclic covering $x \mapsto x^{D / m}$. Different rational expressions for the maximal Darboux coverings are related to each other by Möbius transformations, although the coefficients are cumbersome algebraic numbers. For example, to transform $x^{3}\left(x^{3}+4\right)^{3} / 4\left(2 x^{3}-1\right)^{3}$ to the composition of (3.3) with $x \mapsto x^{2}$, a Möbius transformation is

$$
x \mapsto \sqrt[3]{5-3 \sqrt{3}} \frac{x+\sqrt{3+2 \sqrt{3}}}{x-\sqrt{-3+2 \sqrt{3}}} .
$$

\subsection{Darboux curves for other Schwarz types}

The following lemma allows us to compute all necessary Darboux coverings once Darboux coverings for standard hypergeometric equations (from Section 3.2) are known. For this purpose we usually take $E_{1}$ to be a main representative equation of a chosen Schwarz type, and we usually take $E_{0}$ to be the corresponding standard equation.

Lemma 3.5. Let $E_{0}, E_{1}$ denote two linear differential equations. Suppose that $E_{1}$ is a pullback of $E_{0}$ with respect to a covering $\psi: \mathbb{P}^{1} \rightarrow \mathbb{P}^{1}$. Suppose that $\phi_{0}: D_{0} \rightarrow \mathbb{P}^{1}$ is a Darboux covering for $E_{0}$. Then the fiber product $D_{1}$ of $\psi: \mathbb{P}^{1} \rightarrow \mathbb{P}^{1}$ and $\phi_{0}: D_{0} \rightarrow \mathbb{P}^{1}$ is a Darboux curve for $E_{1}$, and the projection $\phi_{1}: D_{1} \rightarrow \mathbb{P}^{1}$ (to the source of $\psi$ ) is a Darboux covering for $E_{1}$ of the same degree as $\phi_{0}$.

Proof. Let $z$ denote a projective parameter for the $\mathbb{P}^{1}$ below, and let $x$ denote a projective parameter of the other $\mathbb{P}^{1}$, so that the covering $\psi$ corresponds to the extension $\mathbb{C}(x) \supset \mathbb{C}(z)$. Suppose that $u_{0}$ is a Riccati solution for $E_{0}$ which determines $D_{0}$, so that $\mathbb{C}\left(D_{0}\right) \cong \mathbb{C}\left(z, u_{0}\right)$. Then there is a solution $y$ for $E_{0}$ which satisfies $y^{\prime}=u_{0} y$. The logarithmic derivative of the pull-back of $y$ with respect to (A.3) is $u_{0} \psi^{\prime}+\theta^{\prime} / \theta$. It is a Riccati solution for $E_{1}$, and it lies in $\mathbb{C}\left(x, u_{0}\right) \cong \mathbb{C}(x) \otimes_{\mathbb{C}(z)} \mathbb{C}\left(x, u_{0}\right)$ which is the function field for the fiber product of $\psi$ and $\phi_{0}$. Since the degree of the projection to $\mathbb{P}^{1}$ is equal to the degree of $\psi$, the claim follows.

The simplest coverings for Klein pulls-backs $\psi$ from standard hypergeometric equations to other main representatives were first computed in [Sch72]. These Klein coverings are familiar from classical transformations of hypergeometric series (see for instance [AAR99] or [Vid09, Section 5]), except those for the Schwarz types $(1 / 2,1 / 3,2 / 5),(1 / 3,2 / 3,1 / 5)$, $(1 / 3,2 / 5,3 / 5)$. Here we give rational functions that define Klein pulls-backs for their main representatives:

$$
\begin{gathered}
\frac{x^{2}(189-64 x)^{5}}{\left(3584 x^{2}+2457 x-2916\right)^{3}}, \frac{4 x(25 x-9)^{5}}{27(x-1)(125 x+3)^{3}}, \\
\frac{3125 x^{2}(x-1)^{3}(5 x+27)^{5}}{4\left(625 x^{3}-2875 x^{2}+675 x-729\right)^{3}} .
\end{gathered}
$$


TABLE 1. Genus of Darboux curves.

\begin{tabular}{ccccccc}
\hline \multirow{2}{*}{$\begin{array}{c}\text { Denominators } \\
k, \ell, m\end{array}$} & $\begin{array}{c}\text { Schwarz } \\
\text { type }\end{array}$ & $\begin{array}{c}\text { Klein } \\
\text { degree }\end{array}$ & \multicolumn{3}{c}{$g(D)$, when $\operatorname{deg} \gamma: \widetilde{D} \rightarrow D$ is equal to } \\
\cline { 5 - 7 } $2,3,3$ & $(1 / 2,1 / 3,1 / 3)$ & 1 & 0 & 0 & 0 & 0 \\
& $(1 / 3,1 / 3,2 / 3)$ & 2 & 0 & 0 & 1 & 1 \\
$2,3,4$ & $(1 / 2,1 / 3,1 / 4)$ & 1 & 0 & 0 & 0 & 0 \\
& $(2 / 3,1 / 4,1 / 4)$ & 2 & 0 & 1 & 2 & 3 \\
$2,3,5$ & $(1 / 2,1 / 3,1 / 5)$ & 1 & 0 & 0 & 0 & 0 \\
& $(1 / 2,1 / 3,2 / 5)$ & 7 & 0 & 0 & 0 & 0 \\
& $(1 / 2,1 / 5,2 / 5)$ & 3 & 0 & 2 & 2 & 4 \\
& $(1 / 3,1 / 3,2 / 5)$ & 2 & 1 & 1 & 3 & 5 \\
& $(1 / 3,2 / 3,1 / 5)$ & 6 & 1 & 1 & 3 & 5 \\
& $(2 / 3,1 / 5,1 / 5)$ & 2 & 1 & 3 & 5 & 9 \\
& $(1 / 3,2 / 5,3 / 5)$ & 10 & 1 & 3 & 5 & 9 \\
& $(1 / 3,1 / 5,3 / 5)$ & 4 & 1 & 3 & 5 & 9 \\
& $(1 / 5,1 / 5,4 / 5)$ & 6 & 1 & 5 & 7 & 13 \\
& $(2 / 5,2 / 5,2 / 5)$ & 6 & 1 & 5 & 7 & 13 \\
\hline
\end{tabular}

Table 1 shows the genus of all Darboux curves; this information can be computed using Lemma 3.5, part (iii) of Lemma 3.4, and the remarks in Appendices A.7 and A.2. The third column shows the degree of Klein morphism for the main representatives of each Schwarz type. We explicitly compute and utilize Darboux coverings of minimal degree $D / m$.

\subsection{Hypergeometric functions on Darboux curves}

Here we describe technical features of identifying and representing radical functions. First we describe radical solutions of a Fuchsian equation with cyclic monodromy.

LEMMA 3.6. Let C be an algebraic curve, and consider a second-order Fuchsian differential equation (3.1) on C. Suppose that its differential Galois group G is finite and cyclic. Suppose that there are singular points with non-integer local exponent differences. Then:

(i) there exist exactly two independent radical solutions $f_{1}, f_{2}$;

(ii) for any regular singular point $P \in C$ of (3.1) where the exponent difference is not an integer, the local exponents of $f_{1}$ and $f_{2}$ are different.

Proof. Let $K_{P V} \supset \mathbb{C}(C)$ be a Picard-Vessiot extension of the differential equation (3.1), and let $V$ denote its space of solutions in $K_{P V}$. The monodromy group $G$ does not act on $V$ by scalar multiplication, because otherwise the quotient of two independent solutions would be in $\mathbb{C}(C)$. Hence the representation of $G$ on $V$ splits into two irreducible representations. Let $f_{1}, f_{2} \in V$ be generators of the two $G$-invariant subspaces. If $P \in C$ is a point where the exponent difference is not an integer, $f_{1}, f_{2}$ are in different spaces invariant under the local monodromy group. Hence, they have well-defined local exponents, which should be different.

Let $H$ denote a hypergeometric equation. Suppose that its monodromy $G$ is tetrahedral, octahedral, or icosahedral. Let $\phi: D \rightarrow \mathbb{P}^{1}$ denote a Darboux covering for $H$, of degree $d$. 
The monodromy group of the pulled-back equation has the order $|G| / m$. Let $f_{1}, f_{2}$ denote its radical solutions, as described in Lemma 3.6. Their branching points project to singular points of $H$. Let $P$ denote such a projected singularity. The local exponent difference for $H$ at $P$ will be a rational number with the denominator $|G| / m$. The projected functions will have different local exponents at $P$. Hence locally, the projected functions will be two different hypergeometric series at $P$ with different local exponents. In other words, they will be among the 24 Kummer's solutions, mentioned in Appendix A.4. The hypergeometric solutions corresponding to $f_{1}, f_{2}$ will have the form $\theta(z){ }_{2} \mathrm{~F}_{1}(\underset{C}{A, B} \mid \mu(z))$, where $\mu(z) \in$ $\{z, 1-z, z /(z-1), 1 / z, 1 /(1-z), 1-1 / z\}$, and the denominator of $C$ is equal to $|G| / d$. Conversely, such a hypergeometric solution will pull-back to $f_{1}$ or $f_{2}$ if the fiber $z=\varphi(x)$ of $\mu(z)=0$ has non-branching points (that are then singularities of $f_{1}$ or $f_{2}$ ).

The following lemma gives a convenient relation for Darboux evaluations of contiguous algebraic Gauss hypergeometric functions. In particular, it applies to differentiated hypergeometric functions.

LemMA 3.7. Let $F_{1}, F_{2}$ denote two contiguous algebraic (but not rational) Gauss hypergeometric functions. Let $D$ denote their common Darboux curve. Then the pull-back of $F_{1} / F_{2}$ is a rational function on $D$.

Proof. The logarithmic derivative $F_{1}^{\prime} / F_{1}$ is a rational function $D$. Up to a factor in $C(D), F_{1}^{\prime}$ is Gauss hypergeometric function contiguous to $F_{1}$. The contiguous relation among $F_{1}, F_{1}^{\prime}$, and $F_{2}$ has coefficients in $\mathbb{C}(x)$, hence the claim follows.

If we take any pair of contiguous evaluations in Section 2, the quotient of the righthand sides is a rational (rather then radical) function on the Darboux curve, as suggested by Lemma 3.7. This allows us to express other contiguous evaluations conveniently as product of a fixed radical function and some rational function on the Darboux curve.

\section{Computation of Darboux evaluations}

This is an outline of actual computations that gave the list of Darboux evaluations in Section 2. If the Darboux curve has genus zero, computations are straightforward. Sections 4.2 and 4.3 are devoted to difficulties of expressing rational and radical functions on genus one Darboux curves.

\subsection{Computation of Darboux curves and coverings}

Darboux coverings for the three standard Schwarz types are given in Section 3.2. To compute other Darboux coverings and curves, Lemma 3.5 is used. Let us fix an Schwarz type which is not standard, and let $E_{1}$ denote its representative hypergeometric equation as listed in Section 1. Suppose that $\varphi: \mathbb{P}_{z}^{1} \mapsto \mathbb{P}_{Z}^{1}$ is a Darboux covering (of minimal degree) for the corresponding standard hypergeometric equation. Suppose that $\psi: \mathbb{P}_{X}^{1} \mapsto \mathbb{P}_{Z}^{1}$ is a Klein covering for $E_{1}$. Then the Darboux curve is a fiber product of $\varphi$ and $\psi$ by Lemma 3.5. An equation for it is given by the equation $\psi(X)=\phi(z)$.

If the Darboux curve has genus zero, then a parameterization of it immediately gives the Darboux covering. As an example, consider the icosahedral type $(1 / 2,1 / 3,2 / 5)$. We apply Lemma 3.5 with $E_{0}=E(1 / 5,1 / 2,1 / 3)$ and $E_{1}=E(2 / 5,1 / 2,1 / 3)$. By formulas (2.9) and 
(3.6) we obtain the following equation for the Darboux curve:

$$
\frac{X^{2}(189-64 X)^{5}}{\left(3584 X^{2}+2457 X-2916\right)^{3}}=\frac{1728 z\left(z^{2}-11 z-1\right)^{5}}{\left(z^{4}+228 z^{3}+494 z^{2}-228 z+1\right)^{3}} .
$$

This is a rational curve. It can be parameterized by standard algorithms and computer algebra systems such as Maple. The following is a parameterization:

$$
X=\frac{1728 x\left(x^{2}-11 x-1\right)^{5}}{\left(x^{4}+228 x^{3}+494 x^{2}-228 x+1\right)^{3}}, \quad z=\frac{(7 x-1)^{5}}{x^{2}(x+7)^{5}} .
$$

(Recall that parameterizations are unique up to fractional-linear transformations on $\mathbb{P}_{x}^{1}$.) The parametric expression for $X$ gives the Darboux covering for $E(2 / 5,1 / 2,1 / 3)$. We recognize that this is the same Darboux covering (2.9) as for the Schwarz type $(1 / 2,1 / 3,1 / 5)$.

With a computer algebra package and standard algorithms [Kov86, vH94], at hand, it is straightforward to pull-back a hypergeometric equation to a Darboux curve of genus zero, and solve the pulled-back differential equation. In Section 3.4 we characterized the hypergeometric functions to be identified with radical solutions. Since there are only two radical solutions by Lemma 3.6, computer algebra systems should return them. (Otherwise a simplified version of the procedure in Section 4.3 can be considered.)

If the Darboux curve $D$ has genus 1, we first wish to compute a convenient Weierstrass model from the equation $\psi(X)=\varphi(z)$. With such a model at hand, we identify $D$ with the elliptic curve $(D, \mathcal{O})$, where $\mathcal{O}$ denotes the point at infinity. We always seek to have the point $(x, \xi)=(0,0)$ on $D$ above $X=0$, so to allow easy power series verification of the evaluations in (2.19)-(2.38). The Darboux covering is given by the $X$-component of an isomorphism between the elliptic curve and the model $\psi(X)=\varphi(z)$. We wish to express the covering function in such a way that multiplicities of its zeros and poles would be well visible. This is not a straightforward problem, as discussed in Section 4.2. Pull-backs of hypergeometric equations onto elliptic curves and finding radical solutions of those pullbacks are discussed in Section 4.3. In the rest of this section, we derive the four elliptic curves $C_{3}, C_{4}, C_{5}, C_{6}$, introduced in (2.17), (2.23), (2.29), (2.33) as Darboux curves for some icosahedral Schwarz types.

For the Schwarz type $(1 / 3,1 / 3,2 / 5)$, we use Lemma 3.5 with $E_{0}=E(1 / 5,1 / 2,1 / 3)$ and $E_{1}=E(2 / 5,1 / 3,1 / 3)$. This gives the equation $X^{2} / 4(X-1)=\varphi_{1}(z)$ for the Darboux curve. After applying the fractional-linear transformation $F \mapsto F /(F-1)$ to both sides we obtain

$$
\frac{X^{2}}{(X-2)^{2}}=-\frac{1728 z\left(z^{2}-11 z-1\right)^{5}}{\left(z^{2}+1\right)^{2}\left(z^{4}-522 z^{3}-10006 z^{2}+522 z+1\right)^{2}} .
$$

We collect full squares onto the left-hand side and observe that the Darboux curve is isomorphic to the genus-one curve

$$
\widetilde{\xi}^{2}=-1728 z\left(z^{2}-11 z-1\right)
$$

This curve is isomorphic to $C_{3}$ via the isomorphism $(z, \widetilde{\xi}) \mapsto(3 x, 72 \xi)$. The Darboux covering is given by the $X$-component of the isomorphism between $C_{3}$ and (4.3). We have

$$
\frac{X}{X-2}=\frac{72 \xi\left(9 x^{2}-33 x-1\right)^{2}}{\left(9 x^{2}+1\right)\left(81 x^{4}-14094 x^{3}-90054 x^{2}+1566 x+1\right)},
$$


So

$$
\varphi_{3}(x, \xi)=2 /\left(1+\frac{\left(9 x^{2}+1\right)\left(81 x^{4}-14094 x^{3}-90054 x^{2}+1566 x+1\right)}{72 \xi\left(9 x^{2}-33 x-1\right)^{2}}\right) .
$$

Expression (2.18) for the same function is derived by methods of Section 4.2.

For the Schwarz type $(1 / 3,2 / 3,1 / 5)$, we use Lemma 3.5 with $E_{0}=E(2 / 5,1 / 2,1 / 3)$ and $E_{1}=E(4 / 5,1 / 3,1 / 3)$. Then we get the same equation $X^{2} / 4(X-1)=\varphi_{1}(z)$. Hence, the Darboux curve is the same as for the type $(1 / 3,1 / 3,2 / 5)$.

For the Schwarz type $(2 / 3,1 / 5,1 / 5)$, we use Lemma 3.5 with $E_{0}=E(1 / 5,1 / 2,1 / 3)$ and $E_{1}=E(1 / 5,1 / 5,2 / 3)$. This gives the equation $4 X(1-X)=\varphi_{1}(z)$ for the Darboux curve. After applying the fractional-linear transformation $F \mapsto 1-F$ to both sides we obtain

$$
(1-2 X)^{2}=\frac{\left(z^{2}+1\right)^{2}\left(z^{4}-522 z^{3}-10006 z^{2}+522 z+1\right)^{2}}{\left(z^{4}+228 z^{3}+494 z^{2}-228 z+1\right)^{3}} .
$$

We collect full squares to the left-hand side and observe that the Darboux curve is isomorphic to the genus-one curve

$$
\widetilde{\xi}^{2}=z^{4}+228 z^{3}+494 z^{2}-228 z+1
$$

This curve is isomorphic to $C_{4}$ by the isomorphism

$$
(z, \widetilde{\xi}) \mapsto\left(\frac{57 x-5 \xi}{x+25}, \frac{25\left(\xi^{2}-570 \xi-380 x^{2}+248 x+25\right)}{(x+25)^{2}}\right) .
$$

Like with $\varphi_{3}(x, \xi)$, we identify $\varphi_{4}(x, \xi)$ with the $X$-component of the isomorphism between $C_{4}$ and (4.6), and apply methods of Lemma 4.2 to obtain expression (2.24).

For the Schwarz type $(1 / 3,2 / 5,3 / 5)$, we use Lemma 3.5 with $E_{0}=E(2 / 5,1 / 2,1 / 3)$ and $E_{1}=E(2 / 5,2 / 5,2 / 3)$. Then we get the same equation $4 X(1-X)=\varphi_{1}(z)$. Hence, the Darboux curve is the same as for the type $(2 / 3,1 / 5,1 / 5)$.

For the Schwarz type $(1 / 3,1 / 5,3 / 5)$, we use Lemma 3.5 with $E_{0}=E(1 / 5,1 / 2,1 / 3)$ and $E_{1}=E(1 / 5,1 / 3,3 / 5)$. This gives the equation $-64 X /(X-1)(9 X-1)^{3}=\varphi_{1}(z)$ for the Darboux curve. This curve is isomorphic to $C_{5}$, although it is not straightforward to compute a handy isomorphism with current computer algebra packages. The package algcurves of Maple 9.0 can be used to obtain a Weierstrass form and an isomorphism. The isomorphism ought to be simplified using the methods of Section 4.2. Eventually, we obtain an isomorphism given by $z=-2(\xi-3 x)^{2} /(\xi+3 x)(4 x+1)$ and $X=\varphi_{5}(x, \xi)$ as in (2.30).

For the Schwarz type $(1 / 5,1 / 5,4 / 5)$, we use Lemma 3.5 with $E_{0}=E(1 / 5,1 / 2,2 / 5)$ and $E_{1}=E(1 / 5,1 / 5,4 / 5)$. This gives the equation $4 X(1-X)=\varphi_{2}(z)$ for the Darboux curve. After applying the fractional-linear transformation $F \mapsto 1-F$ to both sides we obtain

$$
(1-2 X)^{2}=-\frac{64 x\left(x^{2}-x-1\right)^{5}}{\left(x^{2}+1\right)^{2}\left(x^{4}-22 x^{3}-6 x^{2}+22 x+1\right)^{2}} .
$$

We collect full squares to the left-hand side and easily observe that the Darboux curve is isomorphic $C_{6}$.

For the Schwarz type $(2 / 5,2 / 5,2 / 5)$, we use Lemma 3.5 with $E_{0}=E(2 / 5,1 / 2,1 / 5)$ and $E_{1}=E(2 / 5,2 / 5,2 / 5)$. Then we get the same equation $4 X(1-X)=\varphi_{2}(z)$. Hence, the Darboux curve is the same as for the type $(1 / 5,1 / 5,4 / 5)$. 
TABLE 2. Rational points on elliptic curves.

\begin{tabular}{ccc}
\hline Elliptic curve & Mordell-Weil group & Rational points \\
\hline$C_{3}$ & $\mathbb{Z} / 6 \mathbb{Z}$ & $\mathcal{O},(0,0),\left(-\frac{1}{9}, \frac{5}{9}\right),\left(-\frac{1}{9},-\frac{5}{9}\right),(1,-5),(1,5)$ \\
$C_{4}$ & $\mathbb{Z} \oplus \mathbb{Z} / 2 \mathbb{Z}$ & $\mathcal{O}, \mathcal{O}^{*}=(0,0), A_{n}=n\left(\frac{1}{5}, \frac{3}{5}\right), \widetilde{A}_{n}=-n\left(\frac{1}{5}, \frac{3}{5}\right)$, \\
& & $A_{n}^{*}=n\left(\frac{1}{5}, \frac{3}{5}\right)+\mathcal{O}^{*}, \widetilde{A}_{n}^{*}=-n\left(\frac{1}{5}, \frac{3}{5}\right)+\mathcal{O}^{*}$ \\
$C_{5}$ & $\mathbb{Z} / 4 \mathbb{Z} \oplus \mathbb{Z} / 2 \mathbb{Z}$ & $\mathcal{O},(0,0),\left(-\frac{1}{4},-\frac{3}{4}\right),\left(-\frac{1}{4},-\frac{3}{4}\right)$, \\
& & $(-1,0),\left(-\frac{1}{16}, 0\right),\left(\frac{1}{4},-\frac{5}{4}\right),\left(\frac{1}{4}, \frac{5}{4}\right)$ \\
$C_{6}$ & $\mathbb{Z} / 6 \mathbb{Z}$ & $\mathcal{O},(0,0),(-1,1),(-1,-1),(1,-1),(1,1)$ \\
\hline
\end{tabular}

Note that the elliptic curves $C_{3}, C_{4}, C_{5}, C_{6}$ are defined over $\mathbb{Q}$. It is useful to know rational points on them. Table 2 gives this arithmetic information [Sil86]. It was computed using Maple package Apecs [Con]. Recall that by $\mathcal{O}$ we denote the point at infinity. As we see, only the curve $C_{4}$ has infinitely many rational points. In Table 2 we introduce the notation $\mathcal{O}^{*}, A_{n}, \widetilde{A}_{n}, A_{n}^{*}, \widetilde{A}_{n}^{*}$ (with positive $n \in \mathbb{Z}$ ) for the rational points on $C_{4}$.

\subsection{Representing functions on genus-one curves}

Here we consider the problem of expressing rational functions on elliptic curves. Foremost, we use techniques of this section to compute expressions (2.18), (2.24), (2.30), and (2.34) for Darboux coverings from genus-one Darboux curves. Section 4.3 extends these techniques for computation of expressions on the right-hand sides of (2.19)-(2.22), (2.25)-(2.28), (2.31)(2.32), and (2.35)-(2.38).

A canonical way to represent a rational functions $F$ on a (hyper)elliptic curve $\xi^{2}=G(x)$ (with $G(x) \in \mathbb{C}[x]$ ) is the sum $f_{1}(x)+\xi f_{2}(x)$, with $f_{1}(x), f_{2}(x) \in \mathbb{C}(x)$. This representation suits well algebraic computations, but it gives little geometric information about the function. For example, the principal divisor for a function can be much simpler than the degree of $f_{1}(x)$ and $f_{2}(x)$ may suggest. We would like to have a compact expression that reflects well multiplicities in the principal divisor. We do not give strict definitions or algorithms for an alternative representation. Rather, we give tables of principal divisors on the elliptic curves $C_{3}, C_{4}, C_{5}, C_{6}$, and propose to combine those principal divisors to make the divisor for $F$. The corresponding multiplicative expression in $\mathbb{C}[x, \xi]$-polynomials from our tables will give, up to a constant multiple, a compact expression for $F$ that we seek. We need to compute only finitely many rational and radical functions on elliptic curves, and our tables give enough information for these purposes.

Concretely, we start with Darboux covering $\varphi_{3}(x, \xi)$. Its principal divisor is computed from (4.5) to be the following:

$$
(0,0)+\mathcal{O}+5\left(\frac{11+5 \sqrt{5}}{6}, 0\right)+5\left(\frac{11-5 \sqrt{5}}{6}, 0\right)-3 R_{1}-3 R_{2}-3 R_{3}-3 R_{4}
$$

Here the four points $R_{1}, R_{2}, R_{3}, R_{4}$ are defined by the equations

$$
81 x^{4}+6156 x^{3}+4446 x^{2}-684 x+1=0, \quad 150 \xi=27 x^{3}+1989 x^{2}+741 x-7 .
$$


TABle 3. Principal divisors on $C_{3}$.

\begin{tabular}{cc}
\hline Function & Divisor \\
\hline$\xi$ & $(0,0)+\left(\frac{11+5 \sqrt{5}}{6}, 0\right)+\left(\frac{11-5 \sqrt{5}}{6}, 0\right)-3 \mathcal{O}$ \\
$1+33 x-9 x^{2}$ & $2\left(\frac{11+5 \sqrt{5}}{6}, 0\right)+2\left(\frac{11-5 \sqrt{5}}{6}, 0\right)-4 \mathcal{O}$ \\
$1-9 \xi+54 x$ & $3\left(-\frac{1}{9},-\frac{5}{9}\right)-3 \mathcal{O}$ \\
$1+9 \xi+54 x$ & $3\left(-\frac{1}{9}, \frac{5}{9}\right)-3 \mathcal{O}$ \\
$1+9 x$ & $\left(-\frac{1}{9},-\frac{5}{9}\right)+\left(-\frac{1}{9}, \frac{5}{9}\right)-2 \mathcal{O}$ \\
$\xi+5 x$ & $(0,0)+\left(-\frac{1}{9}, \frac{5}{9}\right)+(1,-5)-3 \mathcal{O}$ \\
$1+21 \xi-117 x+9 x \xi-234 x^{2}$ & $R_{1}+R_{2}+R_{3}+R_{4}+\left(-\frac{1}{9},-\frac{5}{9}\right)-5 \mathcal{O}$ \\
$1-21 \xi-117 x-9 x \xi-234 x^{2}$ & $\widetilde{R}_{1}+\widetilde{R}_{2}+\widetilde{R}_{3}+\widetilde{R}_{4}+\left(-\frac{1}{9}, \frac{5}{9}\right)-5 \mathcal{O}$ \\
\hline
\end{tabular}

Table 3 gives a list of principal divisors on $C_{3}$. For $i \in\{1,2,3,4\}$, let $\widetilde{R}_{i}$ denote the inverse of $R_{i}$ in the group structure of $C_{3}$. Divisor (4.10) can be rewritten as follows:

$$
\begin{aligned}
& \left\{(0,0)+\left(\frac{11+5 \sqrt{5}}{6}, 0\right)+\left(\frac{11-5 \sqrt{5}}{6}, 0\right)-3 \mathcal{O}\right\} \\
& \quad+2\left\{2\left(\frac{11+5 \sqrt{5}}{6}, 0\right)+2\left(\frac{11-5 \sqrt{5}}{6}, 0\right)-4 \mathcal{O}\right\}+\left\{3\left(-\frac{1}{9},-\frac{5}{9}\right)-3 \mathcal{O}\right\} \\
& \quad-3\left\{R_{1}+R_{2}+R_{3}+R_{4}+\left(-\frac{1}{9},-\frac{5}{9}\right)-5 \mathcal{O}\right\} .
\end{aligned}
$$

Observe that each divisor in curled brackets is present in Table 3. We can immediately build the corresponding multiplicative combination of the functions $\xi, 1+33 x-9 x^{2}$, $1-9 \xi+54 x, 1+21 \xi-117 x+9 x \xi-234 x^{2}$. Up to undetermined constant multiple, the multiplicative expression is (2.18). The constant multiple is determined by evaluating the multiplicative expression and (4.5) a convenient point, say $\left(-\frac{1}{9}, \frac{5}{9}\right)$.

As an extra exercise, one may consider the function $1-\varphi_{3}$. Its divisor is computed from (2.18) or (4.5) to be

$$
3 \widetilde{R}_{1}+3 \widetilde{R}_{2}+3 \widetilde{R}_{3}+3 \widetilde{R}_{4}-3 R_{1}-3 R_{2}-3 R_{3}-3 R_{4}
$$

A straightforward combinatorial work suggests the expression

$$
1-\varphi_{3}(x, \xi)=\frac{\left(1-21 \xi-117 x-9 x \xi-234 x^{2}\right)^{3}(1-9 \xi+54 x)}{\left(1+21 \xi-117 x+9 x \xi-234 x^{2}\right)^{3}(1+9 \xi+54 x)} .
$$

Our proposal boils down to building a sufficient table of principal divisors, and combining the known principal divisors to arrive at the principal divisor of a target function. In practice, both things are done in parallel. We start with the functions we wish to express, and compute their divisors. We look at $\mathbb{Q}$-rational points that occur, and use knowledge of the Mordell-Weil group (see Table 2) to foresee and compute suitable $\mathbb{C}[x, \xi]$-polynomials that vanish only on rational points. We may need to distinguish $\mathbb{Q}$-irreducible divisor components of higher degree. For each such divisor component $\Gamma$, we use Gröbner bases to find $\mathbb{C}[x, \xi]$ polynomials of minimal degree that vanish on $\Gamma$ with sufficient multiplicities. We choose 
those polynomials whose divisors enlarge $\Gamma$ minimally or least awkwardly. We look at additional components that occur (usually they are rational points); if they are new, we introduce new polynomials that could compensate the additional components.

For example, consider the $\mathbb{Q}$-irreducible component $R_{1}+R_{2}+R_{3}+R_{4}$ defined by (4.11). A Gröbner basis gives the following quadratic polynomials that vanish on it:

$$
9 x \xi-234 x^{2}+21 \xi-117 x+1, \quad 3 \xi^{2}-2088 x^{2}+150 \xi-744 x+7 .
$$

Other quadratic polynomials are obtained by linear combination. We have chosen the first polynomial in (4.14), and consequently we had to compensate for its additional component $\left(-\frac{1}{9},-\frac{5}{9}\right)$. A reasonable alternative is the quadratic polynomial $\xi^{2}-21 x \xi-150 x^{2}+\xi+$ $25 x$, whose divisor is $R_{1}+R_{2}+R_{3}+R_{4}+(0,0)+(1,-5)-6 \mathcal{O}$.

Next consider the computation of expression (2.24) for the Darboux covering $\varphi_{4}(x, \xi)$. This is the most complicated case, so our description of the computational method reaches deeper refinement level. Let us introduce the functions $N$ and $L$ on the rational points of $C_{4}$ :

$$
\begin{array}{lllll}
N\left(A_{n}\right)=n, & N\left(A_{n}^{*}\right)=n, & N\left(\widetilde{A}_{n}\right)=-n, & N\left(\widetilde{A}_{n}^{*}\right)=-n, & N\left(\mathcal{O}^{*}\right)=0, \\
L\left(A_{n}\right)=0, & L\left(A_{n}^{*}\right)=1, & L\left(\widetilde{A}_{n}\right)=0, & L\left(\widetilde{A}_{n}^{*}\right)=1, & L\left(\mathcal{O}^{*}\right)=1 .
\end{array}
$$

Principal divisors of $\mathbb{C}[x, \xi]$-polynomials have the form $\sum_{j=1}^{n} S_{j}-n \mathcal{O}$. If all points $S_{j}$ are rational, by Lemma A.1 we must have

$$
\sum_{j=1}^{n} N\left(S_{j}\right)=0 \text { and } \sum_{j=1}^{n} L\left(S_{j}\right) \quad \text { even. }
$$

A preliminary expression for $\varphi_{4}(x, \xi)$ is computed by composing the obvious isomorphism between the curves in (4.6) and (4.7) with isomorphism (4.8). The principal divisor of $\varphi_{4}(x, \xi)$ is

$$
(0,0)+\mathcal{O}+5 P_{1}+5 P_{2}-\left(\frac{1}{125}, \frac{57}{625}\right)-(-25,285)-5 Q_{1}-5 Q_{2},
$$

where

$$
P_{1,2}=\left(-\frac{3}{2} \pm \frac{7}{2 \sqrt{5}}, 7 \mp 3 \sqrt{5}\right), \quad Q_{1,2}=\left(-4 \pm \frac{9}{\sqrt{5}},-27 \pm 12 \sqrt{5}\right) .
$$

In the notation of Table 2 , we have $\left(\frac{1}{125}, \frac{57}{625}\right)=A_{5}$ and $(-25,285)=A_{5}^{*}$.

It seems convenient to consider the lines through $P_{1}, P_{2}$ and through $Q_{1}, Q_{2}$. Their equations are $7 \xi=4-30 x$ and $3 \xi=20 x-1$, respectively. The third points on these two lines are, respectively, $\widetilde{A}_{6}^{*}$ and $\widetilde{A}_{4}^{*}$. However, after adding or subtracting extra divisor terms $5 \widetilde{A}_{6}^{*}, 5 \widetilde{A}_{4}^{*}$ in (4.17), it is very cumbersome to compensate them due to (4.16). The $\mathbb{C}[x, \xi]$ polynomials for compensating principal divisors are expected to have very large coefficients.

Rather than introducing the sub-expression $P_{1}+P_{2}+\widetilde{A}_{6}^{*}-3 \mathcal{O}$ in (4.17), we may try to work with the principal divisor $P_{1}+P_{2}+\widetilde{A}_{4}^{*}+\widetilde{A}_{2}-4 \mathcal{O}$. Then $\widetilde{A}_{4}^{*}$ is compensated for automatically. Keeping in mind that the divisors $P_{1}+P_{2}$ and $Q_{1}+Q_{2}$ are equivalent (respectively) to $A_{6}^{*}$ and $A_{4}^{*}$ for the purposes of restrictions (4.16), we work out the following expressions of (4.17) as sums of principal divisors:

$$
\begin{aligned}
& 5\left(P_{1}+P_{2}+\widetilde{A}_{4}^{*}+\widetilde{A}_{2}-4 \mathcal{O}\right)+\left(A_{5}+\widetilde{A}_{5}^{*}+\mathcal{O}^{*}-3 \mathcal{O}\right) \\
& \quad-5\left(Q_{1}+Q_{2}+\widetilde{A}_{4}^{*}-3 \mathcal{O}\right)-\left(5 \widetilde{A}_{2}+2 A_{5}-7 \mathcal{O}\right)-\left(A_{5}^{*}+\widetilde{A}_{5}^{*}-2 \mathcal{O}\right),
\end{aligned}
$$


and

$$
\begin{aligned}
& 5\left(P_{1}+P_{2}+\widetilde{A}_{4}^{*}+\widetilde{A}_{2}-4 \mathcal{O}\right)+\left(A_{10}+\widetilde{A}_{10}^{*}+\mathcal{O}^{*}-3 \mathcal{O}\right) \\
& \quad-5\left(Q_{1}+Q_{2}+\widetilde{A}_{4}^{*}-3 \mathcal{O}\right)-\left(5 \widetilde{A}_{2}+A_{10}-6 \mathcal{O}\right)-\left(A_{5}+A_{5}^{*}+\widetilde{A}_{10}^{*}-3 \mathcal{O}\right) .
\end{aligned}
$$

Now we can build a table of $\mathbb{C}[x, \xi]$-polynomials of the involved principal divisors. Eventually, the two decompositions of (4.17) give the following expressions for $\varphi_{4}(x, \xi)$ :

$$
\frac{8208\left(1-7 \xi+15 x+15 x^{2}\right)^{5}(-5 \xi+57 x)}{(1+3 \xi-20 x)^{5}\left(59375 x^{2} \xi+12350 x \xi+475 \xi-166250 x^{3}-49875 x^{2}-3800 x-19\right)(x+25)},
$$

and, respectively,

$$
\frac{432\left(1-7 \xi+15 x+15 x^{2}\right)^{5}(49495 \xi+292441 x)}{(1+3 \xi-20 x)^{5}\left(2375 \xi^{2}+7400 x \xi+1150 \xi-42000 x^{2}-10200 x-521\right)(2605 \xi+29678 x-475)} .
$$

Compensation of $5 \widetilde{A}_{2}$ looks quite awkward in both formulas.

As the last attempt, we introduce $P_{1}+P_{2}+\widetilde{A}_{5}^{*}+\widetilde{A}_{1}-4 \mathcal{O}$ and $Q_{1}+Q_{2}+\widetilde{A}_{5}^{*}+A_{1}-$ $4 \mathcal{O}$ in (4.17), forgetting divisors of the linear polynomials $7 \xi+30 x-4$ and $3 \xi-20 x+1$. A natural effort to compensate $5 \widetilde{A}_{1}$ and $5 A_{1}$ leads to the following expression decomposition of (4.17):

$$
\begin{aligned}
& 5\left(P_{1}+P_{2}+\widetilde{A}_{5}^{*}+\widetilde{A}_{1}-4 \mathcal{O}\right)+\left(5 A_{1}+\widetilde{A}_{5}-6 \mathcal{O}\right)+\left(2 \mathcal{O}^{*}-2 \mathcal{O}\right) \\
& \quad-5\left(Q_{1}+Q_{2}+\widetilde{A}_{5}^{*}+A_{1}-4 \mathcal{O}\right)-\left(5 \tilde{A}_{1}+A_{5}-6 \mathcal{O}\right)-\left(A_{5}^{*}+\widetilde{A}_{5}+\mathcal{O}^{*}-3 \mathcal{O}\right) .
\end{aligned}
$$

This expression gives formula (2.24). The most convenient principal divisors are listed in Table 4 . The points $R_{1}, R_{2}, R_{3}, R_{4}$ on $C_{4}$ are the points in the fiber $z=1$ of $\varphi_{4}(x, \xi)$.

The principal divisor for $\varphi_{5}(x, \xi)$ on $C_{5}$ turns out to be

$$
(0,0)+\mathcal{O}+5 P_{1}+5 P_{2}-\left(-\frac{1}{4}, \frac{3}{4}\right)-\left(-\frac{1}{4},-\frac{3}{4}\right)-5 Q_{1}-5 Q_{2},
$$

where

$$
P_{1,2}=\left(\frac{-3 \pm \sqrt{5}}{8}, \frac{-5 \pm 3 \sqrt{5}}{8}\right), \quad Q_{1,2}=\left(\frac{7 \pm 3 \sqrt{5}}{8}, \frac{-45 \mp 21 \sqrt{5}}{8}\right) .
$$

As mentioned in Section 4.1, computation of $C_{5}$ and a preliminary expression for $\varphi_{5}(x, \xi)$ was not straightforward. A natural effort leads to the following decomposition of (4.18) into principal ideals:

$$
\begin{aligned}
3 & \left(2\left(\frac{1}{4},-\frac{5}{4}\right)+(0,0)-3 \mathcal{O}\right)+5\left(P_{1}+P_{2}+\left(\frac{1}{4}, \frac{5}{4}\right)-3 \mathcal{O}\right) \\
& -\left(\left(\frac{1}{4}, \frac{5}{4}\right)+\left(\frac{1}{4},-\frac{5}{4}\right)+\left(-\frac{1}{4}, \frac{3}{4}\right)+\left(-\frac{1}{4},-\frac{3}{4}\right)-4 \mathcal{O}\right) \\
& -2\left(2\left(\frac{1}{4}, \frac{5}{4}\right)+(0,0)-3 \mathcal{O}\right)-5\left(Q_{1}+Q_{2}+\left(\frac{1}{4},-\frac{5}{4}\right)-3 \mathcal{O}\right) .
\end{aligned}
$$

This expression gives formula (2.30). The most convenient principal divisors are listed in Table 5. The points $R_{1}, R_{2}, R_{3}, R_{4}$ on $C_{5}$ are the points in the fiber $z=1$ of $\varphi_{5}(x, \xi)$.

The principal divisor for $\varphi_{6}(x, \xi)$ on $C_{5}$ turns out to be

$$
(0,0)+\mathcal{O}+5 P_{1}+5 P_{2}-(1,1)-(-1,1)-5 Q_{1}-5 Q_{2} .
$$


TABle 4. Principal divisors on $C_{4}$.

\begin{tabular}{cccc}
\hline Function & Divisor & Function & Divisor \\
\hline$x$ & $2 \mathcal{O}^{*}-2 \mathcal{O}$ & $1-15 x-5 x^{2}$ & $P_{1}+P_{2}+\widetilde{P}_{1}+\widetilde{P}_{2}-4 \mathcal{O}$ \\
$1-5 x$ & $A_{1}+\widetilde{A}_{1}-2 \mathcal{O}$ & $1-40 x-5 x^{2}$ & $Q_{1}+Q_{2}+\widetilde{Q}_{1}+\widetilde{Q}_{2}-4 \mathcal{O}$ \\
$25+x$ & $A_{5}^{*}+\widetilde{A}_{5}^{*}-2 \mathcal{O}$ & $5-7 \xi-45 x-5 x^{2}$ & $P_{1}+P_{2}+\widetilde{A}_{5}^{*}+\widetilde{A}_{1}-4 \mathcal{O}$ \\
$1-125 x$ & $A_{5}+\widetilde{A}_{5}-2 \mathcal{O}$ & $5+18 \xi-80 x+5 x^{2}$ & $Q_{1}+Q_{2}+\widetilde{A}_{5}^{*}+A_{1}-4 \mathcal{O}$ \\
$5 \xi+57 x$ & $A_{5}^{*}+\widetilde{A}_{5}+\mathcal{O}^{*}-3 \mathcal{O}$ & $1-7 y+15 x+15 x^{2}$ & $P_{1}+P_{2}+\widetilde{A}_{4}^{*}+\widetilde{A}_{2}-4 \mathcal{O}$ \\
$-5 \xi+57 x$ & $\widetilde{A}_{5}^{*}+A_{5}+\mathcal{O}^{*}-3 \mathcal{O}$ & $4-7 \xi-30 x$ & $P_{1}+P_{2}+\widetilde{A}_{6}^{*}-3 \mathcal{O}$ \\
$1+5 \xi+10 x$ & $2 \widetilde{A}_{1}+A_{2}-3 \mathcal{O}$ & $1+3 \xi-20 x$ & $Q_{1}+Q_{2}+\widetilde{A}_{4}^{*}-3 \mathcal{O}$ \\
$1-3 \xi+2 x$ & $A_{2}+A_{2}^{*}+\widetilde{A}_{4}^{*}-3 \mathcal{O}$ & $1-8 \xi+22 x-15 x^{2}$ & $2 A_{1}+A_{2}^{*}+\widetilde{A}_{4}^{*}-4 \mathcal{O}$ \\
$4+21 \xi+41 x$ & $A_{2}+A_{4}^{*}+\widetilde{A}_{6}^{*}-3 \mathcal{O}$ & $4-35 \xi-101 x$ & $A_{1}^{*}+A_{5}+\widetilde{A}_{6}^{*}-3 \mathcal{O}$ \\
$5-3 \xi-34 x$ & $A_{5}^{*}+\widetilde{A}_{4}^{*}+\widetilde{A}_{1}-3 \mathcal{O}$ & $20-7 \xi-79 x$ & $A_{1}+A_{5}^{*}+\widetilde{A}_{6}^{*}-3 \mathcal{O}$ \\
\hline
\end{tabular}

\begin{tabular}{cc}
\hline Function & Divisor \\
\hline $1+50 x-125 \xi^{2}+450 x \xi-500 x^{2}$ & $5 A_{1}+\widetilde{A}_{5}-6 \mathcal{O}$ \\
$1+50 x-125 \xi^{2}-450 x \xi-500 x^{2}$ & $5 \widetilde{A}_{1}+A_{5}-6 \mathcal{O}$ \\
$25-570 \xi+248 x+\xi^{2}-380 x^{2}$ & $R_{1}+R_{2}+R_{3}+R_{4}+2 \widetilde{A}_{5}^{*}-6 \mathcal{O}$ \\
$4+95 \xi+83 x+21 \xi^{2}-475 x \xi+40 x^{2}$ & $R_{1}+R_{2}+R_{3}+R_{4}+\widetilde{A}_{6}^{*}+\widetilde{A}_{4}^{*}-6 \mathcal{O}$ \\
\hline
\end{tabular}

TABLE 5. Divisors on $C_{5}$.

\begin{tabular}{cc}
\hline Function & Divisor \\
\hline$\xi+5 x$ & $2\left(\frac{1}{4},-\frac{5}{4}\right)+(0,0)-3 \mathcal{O}$ \\
$\xi-5 x$ & $2\left(\frac{1}{4}, \frac{5}{4}\right)+(0,0)-3 \mathcal{O}$ \\
$\xi+3 x$ & $2\left(-\frac{1}{4}, \frac{3}{4}\right)+(0,0)-3 \mathcal{O}$ \\
$\xi-3 x$ & $2\left(-\frac{1}{4},-\frac{3}{4}\right)+(0,0)-3 \mathcal{O}$ \\
$1+\xi+x$ & $\left(-\frac{1}{4},-\frac{3}{4}\right)+\left(\frac{1}{4},-\frac{5}{4}\right)+(-1,0)$ \\
$1-2 \xi+6 x$ & $P_{1}+P_{2}+\left(\frac{1}{4}, \frac{5}{4}\right)-3 \mathcal{O}$ \\
$1+12 x+16 x^{2}$ & $P_{1}+P_{2}+\widetilde{P}_{1}+\widetilde{P}_{2}-4 \mathcal{O}$ \\
$1-2 \xi-14 x$ & $Q_{1}+Q_{2}+\left(\frac{1}{4},-\frac{5}{4}\right)-3 \mathcal{O}$ \\
$1-28 x+16 x^{2}$ & $Q_{1}+Q_{2}+\widetilde{Q}_{1}+\widetilde{Q}_{2}-4 \mathcal{O}$ \\
$1+8 \xi-28 x+8 x \xi-104 x^{2}$ & $R_{1}+R_{2}+R_{3}+R_{4}+\left(\frac{1}{4}, \frac{5}{4}\right)-5 \mathcal{O}$ \\
\hline
\end{tabular}


TABLE 6. Divisors on $C_{6}$.

\begin{tabular}{cccc}
\hline Function & Divisor & Function & Divisor \\
\hline$\xi$ & $(0,0)+P_{1}+P_{2}-3 \mathcal{O}$ & $1+x-x^{2}$ & $2 P_{1}+2 P_{2}-4 \mathcal{O}$ \\
$1-\xi$ & $(-1,1)+2(1,1)-3 \mathcal{O}$ & $1-4 x-x^{2}$ & $Q_{1}+Q_{2}+R_{1}+R_{2}-4 \mathcal{O}$ \\
$1+\xi$ & $(-1,-1)+2(1,-1)-3 \mathcal{O}$ & $\xi+2 x+x^{2}$ & $(0,0)+Q_{1}+Q_{2}-3 \mathcal{O}$ \\
$1+\xi+2 x$ & $3(-1,1)-3 \mathcal{O}$ & $1+\xi-2 x$ & $Q_{1}+Q_{2}+(1,1)-3 \mathcal{O}$ \\
$1-\xi+2 x$ & $3(-1,-1)-3 \mathcal{O}$ & $1-\xi-2 x$ & $R_{1}+R_{2}+(1,-1)-3 \mathcal{O}$ \\
\hline
\end{tabular}

where

$$
P_{1,2}=\left(\frac{1 \pm \sqrt{5}}{2}, 0\right), \quad Q_{1,2}=(-2 \pm \sqrt{5},-5 \pm 2 \sqrt{5}) .
$$

We naturally arrive at the following expression for (4.20):

$$
\begin{aligned}
& \left(P_{1}+P_{2}+(0,0)-\mathcal{O}\right)+2\left(2 P_{1}+2 P_{2}-4 \mathcal{O}\right)+2(2(1,1)+(1,-1)-2 \mathcal{O}) \\
& \quad-(3(-1,1)-3 \mathcal{O})-5\left(Q_{1}+Q_{2}+(1,1)-3 \mathcal{O}\right) .
\end{aligned}
$$

This expression gives formula (2.34). The most convenient principal divisors are listed in Table 6. The points $R_{1}, R_{2}$ on $C_{6}$ are in the fiber $z=1$ of $\varphi_{6}(x, \xi)$.

\subsection{Computation of hypergeometric evaluations}

In principle, evaluations (2.19)-(2.22), (2.25)-(2.28), (2.31)-(2.32), and (2.35)-(2.38) are computed by pulling-back their hypergeometric equations onto $C_{3}, C_{4}, C_{5}$, and $C_{6}$, respectively, and finding radical solutions of the pulled-back differential equations. However, standard computer algebra systems do not handle differential equations on higher genus curves.

The pulled-back equations are cumbersome as we will see. On the other hand, their singular points and exponent differences are easy to see, as explained in Appendix A.5. The local exponents tell us possible coefficients in the principal divisors of the radical solutions. The divisors will have coefficients in $\mathbb{Q}$ rather than $\mathbb{Z}$, as described in Appendix A.1. Possible principal divisors are restricted by Lemma A.2 and Lemma 3.6. For simplest hypergeometric equations, we may end up with just two possible principal divisors for radical solutions. Then we can find and check those solutions without computing the pulled-back equation explicitly. Additional contiguous evaluations can be obtained by differentiating known solutions and contiguous relations, while respecting Lemma 3.7 and avoiding explicit computation with the pulled-back equation. It's only if we have several candidates for radical solutions that we may need to compute them and substitute into the pulled-back equation. A single unknown simple zero of a radical solution can be determined by the arithmetic restriction that the principal divisor is invariant under the Galois action of $\overline{\mathbb{Q}}$.

We start with computation of evaluations (2.19)-(2.20). We use Riemann notation (A.6) and consider the icosahedral hypergeometric equation with the solution space

$$
P\left\{\begin{array}{cccc}
0 & 1 & \infty & \\
0 & 0 & -1 / 30 & z \\
2 / 5 & 1 / 3 & 3 / 10 &
\end{array}\right\} .
$$


Its pull-back $z \mapsto \varphi_{3}(x, \xi), y(z) \mapsto Y\left(\varphi_{3}(x, \xi)\right)$ onto $C_{3}$ has the following regular singular points and local exponents:

$$
\left\{\begin{array}{cccccccc}
(0,0) & \mathcal{O} & \left(\frac{11+5 \sqrt{5}}{6}, 0\right) & \left(\frac{11-5 \sqrt{5}}{6}, 0\right) & R_{1} & R_{2} & R_{3} & R_{4} \\
0 & 0 & 0 & 0 & -\frac{1}{10} & -\frac{1}{10} & -\frac{1}{10} & -\frac{1}{10} \\
\frac{2}{5} & \frac{2}{5} & 2 & 2 & \frac{9}{10} & \frac{9}{10} & \frac{9}{10} & \frac{9}{10}
\end{array}\right\} .
$$

Condition (i) of Lemma A.2 leaves only two candidates for the divisors of radical solutions:

$$
\frac{2}{5} \mathcal{O}-\frac{1}{10} R_{1}-\frac{1}{10} R_{2}-\frac{1}{10} R_{3}-\frac{1}{10} R_{4}, \quad \frac{2}{5}(0,0)-\frac{1}{10} R_{1}-\frac{1}{10} R_{2}-\frac{1}{10} R_{3}-\frac{1}{10} R_{4} .
$$

They must represent divisors of the two solutions of Lemma 3.6 and, by identification of local solutions, the hypergeometric series (2.19) and (2.20). Since the point $x=0$ corresponds to $(0,0) \in C_{3}$, the first divisor correspond to the series in (2.19). The right-hand side of that formula is easy to construct from the divisor by using Table 3 and the known value of lefthand side at $x=0$. Similarly, the second divisor in (4.21) implies the identity

$$
\varphi_{3}(x, \xi)^{2 / 5}{ }_{2} \mathrm{~F}_{1}\left(\begin{array}{c}
7 / 10,11 / 30 \\
7 / 5
\end{array} \mid \varphi_{3}(x, \xi)\right)=\frac{x^{1 / 5}(1-9 \xi+54 x)^{1 / 30}}{\left(1+21 \xi-117 x+9 x \xi-234 x^{2}\right)^{1 / 10}} .
$$

This gives formula (2.20). We derived these identities without even computing the pulledback equation on $C_{3}$.

Next we consider computation of evaluations (2.21)-(2.22). The pull-back $z \mapsto \varphi_{3}(x, \xi)$, $y(z) \mapsto Y\left(\varphi_{3}(x, \xi)\right)$ of the icosahedral hypergeometric equation with the solution space

$$
P\left\{\begin{array}{cccc}
0 & 1 & \infty \\
0 & 0 & -1 / 10 & z \\
1 / 5 & 1 / 3 & 17 / 30
\end{array}\right\}
$$

is the following differential equation (with coefficients in a convenient form):

$$
\begin{aligned}
Y^{\prime \prime} & +\frac{3\left(3+47 \xi+1974 x-2051 \xi^{2}+2676 x \xi+54348 x^{2}+33 \xi^{3}-1002 x \xi^{2}-1548 x^{2} \xi\right)}{10 \xi^{2}\left(1+21 \xi-117 x+9 x \xi-234 x^{2}\right)} Y^{\prime} \\
& +\frac{51 \xi(1-9 \xi+54 x)\left(1-21 \xi-117 x-9 x \xi-234 x^{2}\right)}{25 x^{2}(1+9 x)\left(1+21 \xi-117 x+9 x \xi-234 x^{2}\right)^{2}} Y=0
\end{aligned}
$$

As in the previous case above, we know singularities and local exponents of this equation without cumbersome computations. They are

$$
\left\{\begin{array}{cccccc}
(0,0) & \mathcal{O} & R_{1} & R_{2} & R_{3} & R_{4} \\
0 & 0 & -\frac{3}{10} & -\frac{3}{10} & -\frac{3}{10} & -\frac{3}{10} \\
\frac{1}{5} & \frac{1}{5} & \frac{17}{10} & \frac{17}{10} & \frac{17}{10} & \frac{17}{10}
\end{array}\right\}
$$

Condition (i) of Lemma A.2 gives the following candidates for the divisors of radical solutions:

$$
\begin{gathered}
\frac{1}{5} \mathcal{O}+X-\frac{3}{10} R_{1}-\frac{3}{10} R_{2}-\frac{3}{10} R_{3}-\frac{3}{10} R_{4}, \\
\frac{1}{5}(0,0)+Y-\frac{3}{10} R_{1}-\frac{3}{10} R_{2}-t \frac{3}{10} R_{3}-\frac{3}{10} R_{4}
\end{gathered}
$$


Here $X$ and $Y$ are some regular points of (4.22). By condition (ii) and the additional statement of the same lemma, these should be torsion points on $C_{3}$ defined over $\mathbb{Q}$. The possibilities for $X$ and $Y$ are $\left(-\frac{1}{9},-\frac{5}{9}\right),\left(-\frac{1}{9}, \frac{5}{9}\right),(1,-5),(1,5)$. This gives eight possible divisors of a radical solution. For each possibility, one has to construct a radical function (in any form) with that divisor, and to check whether it is a solution of (4.22). Alternatively, candidate solutions can be expanded in power series around $(x, \xi)=(0,0)$ and compared with the hypergeometric series in (2.21) and (2.22). Then one does not have to know the explicit equation (4.22), but has to find enough power series terms of all candidate solutions (so that the right candidates could be selected). It turns out that actual solutions have $X=\left(-\frac{1}{9},-\frac{5}{9}\right)$ and $Y=(1,-5)$. These two solutions and evaluations $(2.21),(2.22)$ are expressed in a convenient form by using methods of Section 4.2.

Next we consider evaluation of (2.25)-(2.26). A pull-back of $E(1 / 5,1 / 5,2 / 3)$ has the following singularities and local exponents:

$$
\left\{\begin{array}{cccccccccc}
\mathcal{O}^{*} & \mathcal{O} & R_{1} & R_{2} & R_{3} & R_{4} & A_{5} & A_{5}^{*} & Q_{1} & Q_{2} \\
0 & 0 & 0 & 0 & 0 & 0 & -\frac{1}{30} & -\frac{1}{30} & -\frac{1}{6} & -\frac{1}{6} \\
\frac{1}{5} & \frac{1}{5} & 2 & 2 & 2 & 2 & \frac{1}{6} & \frac{1}{6} & \frac{5}{6} & \frac{5}{6}
\end{array}\right\} .
$$

Condition (i) of Lemma A.2 gives the following candidates for the divisors on $C_{4}$ of radical solutions:

$$
\begin{aligned}
-\frac{1}{6} Q_{1}-\frac{1}{6} Q_{2}+\frac{1}{6} A_{5}+\frac{1}{6} A_{5}^{*}, & \frac{1}{5} \mathcal{O}+\frac{1}{5}(0,0)-\frac{1}{6} Q_{1}-\frac{1}{6} Q_{2}-\frac{1}{30} A_{5}-\frac{1}{30} A_{5}^{*}, \\
\frac{1}{5} \mathcal{O}-\frac{1}{6} Q_{1}-\frac{1}{6} Q_{2}+\frac{1}{6} A_{5}-\frac{1}{30} A_{5}^{*}, & \frac{1}{5}(0,0)-\frac{1}{6} Q_{1}-\frac{1}{6} Q_{2}-\frac{1}{30} A_{5}+\frac{1}{6} A_{5}^{*}, \\
\frac{1}{5} \mathcal{O}-\frac{1}{6} Q_{1}-\frac{1}{6} Q_{2}-\frac{1}{30} A_{5}+\frac{1}{6} A_{5}^{*}, & \frac{1}{5}(0,0)-\frac{1}{6} Q_{1}-\frac{1}{6} Q_{2}+\frac{1}{6} A_{5}-\frac{1}{30} A_{5}^{*} .
\end{aligned}
$$

The candidate divisors are grouped into possible pairs for a basis of actual solutions, following Lemma 3.6. The divisors in the first pair do not satisfy condition (ii) of Lemma A.2. To decide the right pair, we take one divisor from each of the latter two pairs, construct an expression for a corresponding radical function, and compare its power series around $(0,0)$. It turns out that the last pair is the right one. Once we have the right divisors for (2.25) and (2.26), we can proceed similarly as in the pervious cases. Application of the methods of Section 4.2 may require some combinatorial creativeness. For example, here is a convenient splitting of the right divisor for (2.25):

$$
\frac{1}{6}\left(\widetilde{A}_{4}^{*}+A_{5}^{*}+\widetilde{A}_{1}-3 \mathcal{O}\right)-\frac{1}{6}\left(Q_{1}+Q_{2}+\widetilde{A}_{4}^{*}-3 \mathcal{O}\right)-\frac{1}{30}\left(5 \widetilde{A}_{1}+A_{5}-6 \mathcal{O}\right) .
$$

Now we consider evaluation of (2.27)-(2.28). We comment only on the most complicated step of choosing the divisors of actual solutions of the pull-back of $E(2 / 5,3 / 5,1 / 3)$. The singularities and local exponents are as follows:

$$
\left\{\begin{array}{cccccccc}
\mathcal{O}^{*} & \mathcal{O} & P_{1} & P_{2} & A_{5} & A_{5}^{*} & Q_{1} & Q_{2} \\
0 & 0 & 0 & 0 & -\frac{1}{6} & -\frac{1}{6} & -\frac{5}{6} & -\frac{5}{6} \\
\frac{2}{5} & \frac{2}{5} & 2 & 2 & \frac{13}{30} & \frac{13}{30} & \frac{13}{6} & \frac{13}{6}
\end{array}\right\} .
$$


Condition (i) of Lemma A.2 gives the following candidates for the divisors of radical solutions:

$$
\begin{gathered}
-\frac{1}{6} A_{5}-\frac{1}{6} A_{5}^{*}-\frac{5}{6} Q_{1}-\frac{5}{6} Q_{2}+2 P_{1}, \quad-\frac{1}{6} A_{5}-\frac{1}{6} A_{5}^{*}-\frac{5}{6} Q_{1}-\frac{5}{6} Q_{2}+2 P_{2}, \\
-\frac{1}{6} A_{5}-\frac{1}{6} A_{5}^{*}-\frac{5}{6} Q_{1}-\frac{5}{6} Q_{2}+X+Y, \quad X+Y \in\left\{A_{5}, A_{5}^{*}\right\} \\
\frac{2}{5} \mathcal{O}+\frac{2}{5} \mathcal{O}^{*}+\frac{13}{30} A_{5}+\frac{13}{30} A_{5}^{*}-\frac{5}{6} Q_{1}-\frac{5}{6} Q_{2}, \\
\frac{2}{5} \mathcal{O}+\frac{13}{30} A_{5}-\frac{1}{6} A_{5}^{*}-\frac{5}{6} Q_{1}-\frac{5}{6} Q_{2}+X, \quad X \in\left\{A_{2}, A_{2}^{*}\right\} \\
\frac{2}{5} \mathcal{O}-\frac{1}{6} A_{5}+\frac{13}{30} A_{5}^{*}-\frac{5}{6} Q_{1}-\frac{5}{6} Q_{2}+X, \quad X \in\left\{A_{2}, A_{2}^{*}\right\}, \\
\frac{2}{5} \mathcal{O}^{*}+\frac{13}{30} A_{5}-\frac{1}{6} A_{5}^{*}-\frac{5}{6} Q_{1}-\frac{5}{6} Q_{2}+X, \quad X \in\left\{A_{2}, A_{2}^{*}\right\} \\
\frac{2}{5} \mathcal{O}^{*}-\frac{1}{6} A_{5}+\frac{13}{30} A_{5}^{*}-\frac{5}{6} Q_{1}-\frac{5}{6} Q_{2}+X, \quad X \in\left\{A_{2}, A_{2}^{*}\right\} .
\end{gathered}
$$

Here the restrictions on the additional points $X, Y$ follow from condition (ii) and the additional statement of Lemma A.2. The first three possibilities can be paired only with the fourth divisor as (divisors of) functions $f_{1}, f_{2}$ of Lemma 3.6. To refute them, one has to check only that a function with the fourth divisor (as the principal divisor) is not a solution of the pulled-back equation. Other possibilities have to be paired and checked as we did for equation (4.22). The right divisors are

$$
\frac{2}{5} \mathcal{O}+\frac{13}{30} A_{5}-\frac{1}{6} A_{5}^{*}-\frac{5}{6} Q_{1}-\frac{5}{6} Q_{2}+A_{2}^{*}, \quad \frac{2}{5} \mathcal{O}^{*}-\frac{1}{6} A_{5}+\frac{13}{30} A_{5}^{*}-\frac{5}{6} Q_{1}-\frac{5}{6} Q_{2}+A_{2} .
$$

Now we can compute (2.27) and (2.28) as in the previous cases.

Computation of (2.31)-(2.32), (2.35)-(2.38) is similar and no more complicated. First we find singularities and local exponents of the pulled-back equation; make a list of possible divisors for radical solutions of the pull-back; use Lemma 3.6 to make a short divisors list for necessary check; find radical functions for the candidate divisors from the short list; compare their power series around $(0,0)$ with the expansions of the starting hypergeometric series; take the right divisors and find a convenient expression for their functions using methods of Section 4.2.

Differentiation and computation of contiguous evaluations to (2.19)-(2.38) quickly leads to large expressions. For example, an expression contiguous to (2.19) is

$$
\begin{aligned}
& { }_{2} \mathrm{~F}_{1}\left(\begin{array}{c|c}
3 / 10,29 / 30 & \varphi_{3}(x, \xi) \\
3 / 5 & { }^{2}
\end{array}\right) \\
& =\frac{\left(1+21 \xi-117 x+9 x \xi-234 x^{2}\right)^{9 / 10}(1+9 x)^{2}\left(1+198 x-99 x^{2}\right)}{(1-9 \xi+54 x)^{29 / 30}\left(1-21 \xi-117 x-9 x \xi-234 x^{2}\right)^{2}} .
\end{aligned}
$$

The divisor for this function on $C_{3}$ is

$$
\frac{9}{10} R_{1}+\frac{9}{10} R_{2}+\frac{9}{10} R_{3}+\frac{9}{10} R_{4}+S_{1}+S_{2}+\widetilde{S}_{1}+\widetilde{S}_{2}+\frac{2}{5} \mathcal{O}-2 \widetilde{R}_{1}-2 \widetilde{R}_{2}-2 \widetilde{R}_{3}-2 \widetilde{R}_{4},
$$

where $S_{1}, S_{2}, \widetilde{S}_{1}, \widetilde{S}_{2}$ are the points with the $x$-coordinate equal to $1 \pm 10 / 3 \sqrt{11}$. For the least painful computation of contiguous expressions, keep in mind that the quotient of contiguous hypergeometric functions is a rational function on a Darboux curve, by Lemma 3.7. In particular, the above expression should be viewed as $G(x, \xi) \times{ }_{2} \mathrm{~F}_{1}\left(\begin{array}{c}3 / 10,-1 / 30 \\ 3 / 5\end{array} \mid \varphi_{3}\right)$ with $G(x, \xi) \in \mathbb{C}\left(C_{3}\right)$. To compute $G(x, \xi)$, only divisors with integer (rather than $\mathbb{Q}$ ) coefficients have to be considered as in Section 4.2. 
Acknowledgements. This work was supported by the Dutch NWO project 613-06-565, by the ESF NOG-project, and by the JSPS grant number 20740075.

\section{A. Appendix}

Here we recall relevant standard definitions and facts. This material is widely known, but rarely presented in a way which is convenient for our purposes. For similar introductions, we refer to [Ber04, Beu07, vdW02].

\section{A.1. Algebraic curves}

For general theory of algebraic curves we refer to [Ful69] or to [Sha74]. We assume algebraic curves to be reduced, irreducible, smooth, complete (or projective), defined over $\mathbb{C}$. In particular, the projective line $\mathbb{P}^{1}$ is $\mathbb{C} \cup\{\infty\}$ set-theoretically. All curves in this paper are defined over $\mathbb{Q}$, but we do not consider arithmetic properties here.

Let $C$ denote an algebraic curve. We denote the field of rational functions on $C$ by $\mathbb{C}(C)$. It can be generated by two functions, since $C$ is birationally isomorphic to a (possibly singular) curve in $\mathbb{P}^{2}$. The function field $\mathbb{C}\left(\mathbb{P}^{1}\right)$ is generated by one function; such a generator is called a rational parameter.

If $P \in C$ and $f \in \mathbb{C}(C)$, then $\operatorname{ord}_{P}(f)$ denotes the valuation of $f$ at $P$. If negative, this is the order of a pole of $f$ at $P$; otherwise this is the vanishing order of $f$ at $P$. A local parameter at $P$ is a function $t_{P} \in \mathbb{C}(C)$ such that $\operatorname{ord}_{P}\left(t_{P}\right)=1$. For example, if $C$ is the projective line, then $x-\alpha$ is a local parameter at the point $x=\alpha$, and $1 / x$ is a local parameter at $x=\infty$.

A divisor on $C$ is a finite formal sum $\sum_{P \in C} a_{P} P$, with $a_{P} \in \mathbb{Z}$. The degree of such a divisor is the integer $\sum_{P \in C} a_{P}$. The divisors form a commutative group under addition. For a function $f \in \mathbb{C}(C)$ we have its principal divisor $\sum_{P \in C} \operatorname{ord}_{P}(f) P$, which has degree zero. Principal divisors form a subgroup of degree zero divisors. The quotient of these two groups is a Picard group of $C$; it is denoted by $\operatorname{Pic}(C)$. For example, $\operatorname{Pic}\left(\mathbb{P}^{1}\right)$ is the trivial group because all degree-zero divisors on $\mathbb{P}^{1}$ are principal.

Explicit curves in this paper have either genus zero (i.e. isomorphic to $\mathbb{P}^{1}$ ) or genus one. Let $E$ denote a curve of genus one. It can be represented in a Weierstrass form $\xi^{2}=G_{3}(x)$, where $G_{3}(x)$ is a cubic polynomial in $\mathbb{C}[x]$. The point at infinity in this model by $\mathcal{O}$. The Picard group of $E$ is isomorphic (set-theoretically) to $E$ itself. As usual, we identify a point $P \in E$ with the element of $\operatorname{Pic}(E)$ represented by the divisor $P-\mathcal{O}$. Then the additive group law on $E$ can be given by the known chord-and-tangent method. In particular, if three points of $E$ lie on one line of $\mathbb{P}^{2}$, they add up to the neutral element $\mathcal{O}$. The curve $E$ with this group law is an elliptic curve $(E, \mathcal{O})$. Recall that a torsion point on $E$ is a point of finite order.

Lemma A.1. Let $(E, \mathcal{O})$ denote an elliptic curve, and let $T=\sum_{P \in E} a_{P} P$ be a divisor on $E$. Then $T$ is a principal divisor if and only if $\sum_{P \in E} a_{P} P=\mathcal{O}$ in the additive group of $(E, \mathcal{O})$.

Proof. Follows from the specified identification of $(E, \mathcal{O})$ with $\operatorname{Pic}(E)$. 
We also consider radical functions on $C$, that is, products of $\mathbf{Q}$-powers of functions from $\mathbb{C}(C)$. These are multi-valued functions, but their branching points are poles or zeros with finitely many complex branches coming together. Valuations of those functions are well defined at any point, and have values in $\mathbb{Q}$ at the branching points. Accordingly, we consider their principal divisors $\sum_{P \in C} a_{P} P$ with coefficients $a_{P} \in \mathbb{Q}$.

LemmA A.2. Let $(E, \mathcal{O})$ denote an elliptic curve, and let $T=\sum_{P \in E} a_{P} P$ be a divisor with coefficients in $\mathbb{Q}$. Then $T$ is the principal divisor for a radical function if and only if the following conditions hold:

(i) the degree $\sum_{P \in E} a_{P}$ is zero;

(ii) let $\sum_{P \in E} \widetilde{a}_{P} P$ be an integer multiple of $T$ such that all coefficients $\tilde{a}_{P}$ are integers; then, in the additive group of $E$, the point $\sum_{P \in C} \tilde{a}_{P} P$ must be a torsion point.

Proof. Under these conditions, an integer factor $n T$ of $T$ would be a principle divisor with integer coefficients. Then $T$ is a divisor of $G^{1 / n}$ for some $G \in \mathbb{C}(E)$.

On the other hand, if $T$ is a divisor of a radical function $f$, then an integer power $f^{n}$ is a rational function. The divisor $n T$ sums up to $\mathcal{O}$ by Lemma A.1. Other integer factors of $T$ with integral coefficients may sum up to a torsion point.

\section{A.2. Finite coverings and pull-back transformations}

Consider a finite covering $\phi: C \rightarrow D$ from $C$ to other algebraic curve $D$. It induces an algebraic field extension $\mathbb{C}(C) \supset \mathbb{C}(D)$. We denote the degree of $\phi$ by deg $\phi$. The genus $g(C)$ and $g(D)$ of both curves and branching data are related by the Hurwitz formula:

$$
2 g(C)-2=(2 g(D)-2) \operatorname{deg} \phi+\sum_{P \in C}\left(r_{P}-1\right) .
$$

Here $r_{P}$ is the branching order at $P$. It is equal to $\operatorname{ord}_{P}\left(t_{\phi(P)} \circ \phi\right)$.

Let $C$ denote an algebraic curve. Suppose that the function field $\mathbb{C}(C)$ of $C$ is generated by functions $x, \xi$. We define a pull-back transformation of hypergeometric equation (1.1) with respect to a finite covering $\phi: C \rightarrow \mathbb{P}^{1}$ as follows. Let $z$ denote a rational parameter for $\mathbb{P}^{1}$. Then a pull-back of (1.1) with respect to $\phi$ is a differential equation defined by transformation:

$$
z \longmapsto \phi(x, \xi), \quad y(z) \longmapsto Y(x, \xi)=\theta(x, \xi) y(\phi(x, \xi)) .
$$

Here $\theta(x, \xi)$ is a radical function. Note that such a function has the property that its logarithmic derivative $\theta^{\prime}(x, \xi) / \theta(x, \xi)$ is in $\mathbb{C}(C)$. We use the derivation on $\mathbb{C}(C)$ that extends the usual derivative on $\mathbb{C}(x)$. If $C \cong \mathbb{P}^{1}$, we assume that $x$ is a rational parameter of $C$ (and $\xi$ is not used). Then transformation (A.2) is the following:

$$
z \longmapsto \phi(x), \quad y(z) \longmapsto Y(x)=\theta(x) y(\phi(x)) .
$$

\section{A.3. Differential Galois theory}

A differential field $K$ is a field with a derivation, i.e. a map $D: K \rightarrow K$ which satisfies $D(a+b)=D(a)+D(b)$ and the Leibnitz rule $D(a b)=a D(b)+b D(a)$. One usually denotes $D(a)$ by $a^{\prime}$. An extension of the differential field $K$ is a differential field $L$ which 
contains $K$ and whose derivation extends the derivation of $K$. The basic example of a differential field is the field $\mathbb{C}(z)$ of rational functions on $\mathbb{P}^{1}$ with the usual derivation. Other example is the field $\mathbb{C}(C)$ of rational functions on an algebraic curve. To give a derivation on $\mathbb{C}(C)$ one can consider a finite covering $\phi: C \rightarrow \mathbb{P}^{1}$ and the corresponding unique extension of the usual derivation of $\mathbb{C}(z)$.

Fix a differential field $K$ and consider a linear homogeneous differential equation (3.1). Solutions in any extension of $K$ form a linear space over the constant field $\left\{a \in K \mid a^{\prime}=0\right\}$. The dimension of the solution space is at most $n$. A Picard-Vessiot extension $K_{P V} \supset K$ for (3.1) is, roughly speaking, a minimal extension of differential fields such that the solutions of (3.1) in $K_{P V}$ form a linear space of dimension $n$. The differential Galois group $G$ of (3.1) is the group of automorphisms of $K_{P V}$ that fix the elements of $K$. The action of $G$ on the $n$-dimensional space of solutions in $K_{P V}$ gives a faithful $n$-dimensional representation of $G$. Therefore the differential Galois group $G$ is usually considered as an algebraic subgroup of $\mathrm{GL}(n, \mathbb{C})$.

In Section 3, we utilize the Riccati equation associated with (3.1). Solutions for the Riccati equation are precisely the logarithmic derivatives $y^{\prime} / y$ of solutions for (3.1). Explicitly, the Riccati equation for (3.1) with $n=2$ is $u^{\prime}+u^{2}+a_{1} u+a_{0}=0$. Rational or algebraic solutions of the Riccati equation are important [Kov86] in finding 'closed-form' solutions of the original equation (3.1). We refer to algebraic solutions of the Riccati equation in our working definition of Darboux curves.

Suppose that hypergeometric equation (1.1) has a finite monodromy $G$. Then the differential Galois group is isomorphic to $G$. (In general, the differential Galois group of a Fuchsian equation is isomorphic to the Zariski closure of a representation of the monodromy group.) A Picard-Vessiot extension $K_{P V} \supset \mathbb{C}(z)$ is a finite Galois extension, and the usual Galois group is isomorphic to $G$ as well. If $y(z) \in K_{P V}$ is a solution of (1.1), then $K_{P V}=\mathbb{C}(z, y)$.

In most papers on differential Galois theory, second-order differential equations are normalized to the form $y(z)^{\prime \prime}=r(z) y(z)$, with $r(z) \in \mathbb{C}(z)$. Hypergeometric equation (1.1) can be normalized by the transformation

$$
y(z) \longmapsto z^{\left(e_{0}-1\right) / 2}(1-z)^{\left(e_{1}-1\right) / 2} y(z) .
$$

The normalized equation is

$$
\frac{d^{2} y(z)}{d z^{2}}=\left(\frac{e_{1}^{2}-1}{4(z-1)^{2}}+\frac{e_{0}^{2}-1}{4 z^{2}}+\frac{1+e_{\infty}^{2}-e_{0}^{2}-e_{1}^{2}}{4 z(z-1)}\right) y(x) .
$$

If the monodromy $G$ of a hypergeometric equation is isomorphic to $A_{4}, S_{4}$ or $A_{5}$, then the differential Galois group of the normalized equation (A.5) is $G \times\{1,-1\}$. This does not change facts that are important to us. The algebraic degree of Riccati solutions for (1.1) is the same as of Riccati solutions for (A.5). 


\section{A.4. Hypergeometric equations}

The singularities and local exponents of hypergeometric equation (1.1) are conveniently revealed in Riemann's $P$-notation:

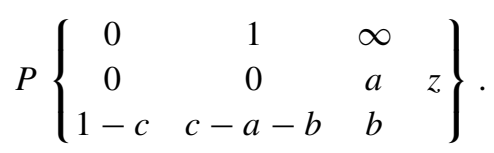

As we see, the first row indicates the regular singular points, and the other rows contain the local exponents and the variable $z$. A general basis of solutions for (1.1) is

$$
{ }_{2} \mathrm{~F}_{1}\left(\begin{array}{c|c}
a, b & z \\
c & z
\end{array}\right), \quad z^{1-c}{ }_{2} \mathrm{~F}_{1}\left(\begin{array}{c|c}
a+1-c, b+1-c & z \\
2-c & -c
\end{array}\right.
$$

where

$$
{ }_{2} \mathrm{~F}_{1}\left(\begin{array}{c|c}
a, b & z \\
c & z
\end{array}\right):=1+\frac{a b}{c \cdot 1 !} z+\frac{a(a+1) b(b+1)}{c(c+1) \cdot 2 !} z^{2}+\cdots
$$

is the Gauss hypergeometric series.

The exponent differences at the three singularities are obviously $e_{0}=1-c, e_{1}=c-$ $a-b$, and $e_{\infty}=a-b$. These linear expressions in $a, b, c$ can be inverted, so that an ordered sequence of exponent differences determines the hypergeometric equation. If we permute the exponent differences $e_{0}, e_{1}, e_{\infty}$ or multiply some of them by -1 , we get hypergeometric equations related by well-known fractional-linear transformations [AAR99]. In general, there are 24 hypergeometric equations related in this way, and they share the same (up to radical factors and fractional-linear change of the independent variable) 24 hypergeometric Kummer's solutions.

\section{A.5. Fuchsian equations}

All differential equations that we explicitly consider are Fuchsian equations. These equations have only regular singular points. For equation (3.1) this means the following: if $K=\mathbb{C}(C)$ for an algebraic curve $C$, then for any point $P \in C$ and for $i=1, \ldots, n$ we must have $\operatorname{ord}_{P}\left(a_{i}\right) \geq(n-i)\left(\operatorname{ord}_{P}\left(t_{P}^{\prime}\right)-1\right)$, where $t_{P}$ is a local parameter at $P$. Local exponents at $P$ are defined as follows: substitute $y=t_{P}^{\mu}$ into the Fuchsian equation and consider the terms to the power $\mu+n\left(\left(\operatorname{ord}_{P}\left(t_{P}^{\prime}\right)-1\right)\right.$ of $t_{P}$ as an equation in $\mu$. The roots of that equation are precisely the local exponents. The local exponents at regular points are equal to $0,1, \ldots, n-1$.

In general, singularities and local exponents do not determine a Fuchsian equation uniquely. Hence, we cannot always use the $P$-notation for general Fuchsian equations. However, in Section 4.3 we write down arrays of singularities and local exponents similar to (A.6).

\section{A.6. Contiguous relations of Gauss hypergeometric functions}

Two Gauss hypergeometric functions are called contiguous (or associated in [Erd53]) if they have the same argument $z$ and their parameters $a, b$ and $c$ differ respectively by integers. As is known [AAR99, Section 2.5], for any three contiguous ${ }_{2} \mathrm{~F}_{1}$ functions there is a contiguous 
relation, which is a linear relation between the three functions where the coefficients are rational functions in the parameters $a, b, c$ and the argument $z$. A straightforward (although not efficient) method to compute a contiguous expression for ${ }_{2} \mathrm{~F}_{1}\left(\begin{array}{c}a+k, b+\ell \\ c+m\end{array} \mid z\right)$ in terms of ${ }_{2} \mathrm{~F}_{1}(\underset{c}{a+1, b} \mid z)$ and ${ }_{2} \mathrm{~F}_{1}(\underset{c}{a, b} \mid z)$ is the following. By using the contiguous relations

$$
\begin{gathered}
b_{2} \mathrm{~F}_{1}\left(\begin{array}{c|c}
a, b+1 & z \\
c & z
\end{array}\right)(b-a)_{2} \mathrm{~F}_{1}\left(\begin{array}{c}
a, b \\
c
\end{array} \mid z\right)+a_{2} \mathrm{~F}_{1}\left(\begin{array}{c}
a+1, b \mid z \\
c
\end{array}\right), \\
(c-1)_{2} \mathrm{~F}_{1}\left(\begin{array}{c}
a, b \\
c-1
\end{array} \mid z\right)=(c-a-1)_{2} \mathrm{~F}_{1}\left(\begin{array}{c}
a, b \\
c
\end{array} \mid z\right)+a_{2} \mathrm{~F}_{1}\left(\begin{array}{c}
a+1, b \mid z \\
c
\end{array}\right),
\end{gathered}
$$

one eliminates the shifts in $b$ and $c$, and then by using the contiguous relation

$$
\begin{aligned}
& a(1-z){ }_{2} \mathrm{~F}_{1}\left(\begin{array}{c}
a+1, b \\
c
\end{array} \mid z\right) \\
& \quad=(2 a-c-a z+b z)_{2} \mathrm{~F}_{1}\left(\begin{array}{c}
a, b \\
c
\end{array} \mid z\right)+(c-a)_{2} \mathrm{~F}_{1}\left(\begin{array}{c}
a-1, b \\
c
\end{array}\right)
\end{aligned}
$$

one obtains an expression with two contiguous terms. Effective computation of contiguous relations is considered in [Vid03, Tak89]. They can be computed in $O(\log (\max (k, l, m))$ steps, but complexity of expressions in each such step grows exponentially, and the output is $O(\max (k, l, m))$.

One can rewrite contiguity conditions in terms of local exponent differences at $z=0$, $1, \infty$ for the hypergeometric equation, since the parameters $a, b, c$ determine the exponent differences and vice versa (if the sign of exponent differences is taken into account). The main hypergeometric solutions (A.7) of two hypergeometric equations (1.1) are contiguous if for each $X \in\{0,1, \infty\}$ the difference of signed exponent differences at $X$ of the two equations is an integer, and the sum of the three integer differences is even. Two hypergeometric equations have solutions contiguous to each other (or, equivalently, they have the same Schwarz type) if one can choose a permutation of exponent differences and their sign in such a way that the just described situation occurs.

For example, the parameters $e_{0}, e_{1}, e_{\infty}$ of hypergeometric equations of the Schwarz type $(1 / 3,1 / 3,2 / 3)$ are characterized as follows: they are rational numbers, their denominators are equal to 3 , and the sum of their numerators is even.

\section{A.7. Fiber products of curves}

Let $C_{1}$ and $C_{2}$ denote two curves over $\mathbb{C}$. Let $\phi_{1}: C_{1} \rightarrow \mathbb{P}^{1}$ and $\phi_{2}: C_{2} \rightarrow \mathbb{P}^{1}$ be two finite coverings of degree $m$ and $n$, respectively. The fiber product of $\phi_{1}: C_{1} \rightarrow \mathbb{P}^{1}$ and $\phi_{2}: C_{2} \rightarrow \mathbb{P}^{1}$ is a curve $B$ with two coverings $\psi_{1}: B \rightarrow C_{1}$ and $\psi_{2}: B \rightarrow C_{2}$ such that $\phi_{1} \circ \psi_{1}=\phi_{2} \circ \psi_{2}$, and for any other curve $\widetilde{B}$ with coverings $\widetilde{\psi}_{1}: \widetilde{B} \rightarrow C_{1}$ and $\widetilde{\psi}_{2}: \widetilde{B} \rightarrow C_{2}$ satisfying $\phi_{1} \circ \widetilde{\psi}_{1}=\phi_{2} \circ \widetilde{\psi}_{2}$ there is a unique covering $\xi: \widetilde{B} \rightarrow B$ such that $\widetilde{\psi}_{1}=\psi_{1} \circ \xi$ and 
$\widetilde{\psi}_{2}=\psi_{2} \circ \xi$. Then the following diagram commutes:

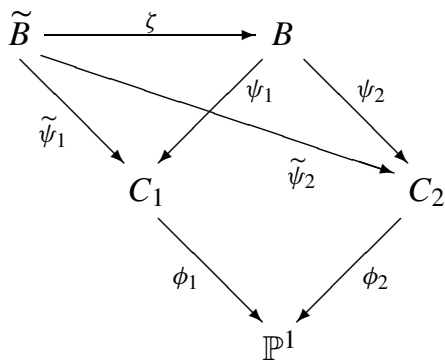

We have $\operatorname{deg} \psi_{1}=\operatorname{deg} \phi_{2}$ and $\operatorname{deg} \psi_{2}=\operatorname{deg} \phi_{1}$. On the level for function fields, we have $\mathbb{C}(B)=\mathbb{C}\left(C_{1}\right) \otimes_{\mathbf{C}\left(\mathbb{P}^{1}\right)} \mathbb{C}\left(C_{2}\right)$.

A birational model for $B$ is the curve on $C_{1} \times C_{2}$ of those points $\left(X_{1}, X_{2}\right) \in C_{1} \times C_{2}$ which satisfy $\varphi_{1}\left(X_{1}\right)=\varphi_{2}\left(X_{2}\right)$. This is a singular model in general. A singular point corresponds to a pair $\left(X_{1}, X_{2}\right) \in B$ such that $X_{1}$ and $X_{2}$ have branching indices $r_{1}>1$, $r_{2}>1$, respectively (with respect to $\phi_{1}$ and $\phi_{2}$ ). Such a singularity is of type $x_{1}^{r_{1}}-x_{2}^{r_{2}}$; by resolving it we get $\operatorname{gcd}\left(r_{1}, r_{2}\right)$ points that correspond to $\left(X_{1}, X_{2}\right)$ on a non-singular model for $B$. This information allows us to compute the branching data for the projections $\psi: B \rightarrow C_{1}$ and $\psi: B \rightarrow C_{2}$ and the genus of $B$. For example, if $X_{1} \in C_{1}$ has branching index $r_{1}$ (with respect to $\left.\phi_{1}\right)$, and the branching data of $\phi_{2}$ above $\phi_{1}\left(X_{1}\right)$ is $a_{1}+\cdots+a_{k}$, then the branching data for $\psi_{1}$ above $X_{1}$ is the following: $\operatorname{gcd}\left(a_{1}, r_{1}\right) * \operatorname{lcm}\left(a_{1}, r_{1}\right) / r_{1}+\cdots+\operatorname{gcd}\left(a_{k}, r_{1}\right) *$ $\operatorname{lcm}\left(a_{k}, r_{1}\right) / r_{1}$.

\section{REFERENCES}

[AAR99] G. E. Andrews, R. Askey, and R. Roy. Special Functions. Cambridge University Press, Cambridge, 1999.

[BD79] F. Baldassarri and B. Dwork. On second order linear differential equations with algebraic solutions. Amer. J. Math. 101 (1979), 42-76.

[Ber04] M. Berkenbosch. Algorithms and Moduli spaces for Differential Equations. PhD Thesis, University of Groningen, 2004.

[Beu07] F. Beukers. Gauss' hypergeometric function. In W. Abikoff et al. (ed.), The Mathematical Legacy of Wilhelm Magnus: Groups, geometry and special functions (Contemporary Mathematics, 169). American Mathematical Society, Providence, RI, 2007, pp. 29-43.

[Bou98] A. Boulanger. Contribution a l'etude des equations lineaires homogenes integrables algebriquement. J. Ecole Polytech. 4 (1898), 1-122.

[Bri77] F. Brioschi. La théorie des formes dans l'intégration des équations différentielles lineéaires du second ordre. Math. Ann. 11 (1877), 401-411.

[BvHW03] M. Berkenbosch, M. van Hoeij and J.-A. Weil. Recent algorithms for solving second order differential equations. Technical report, 2003. Available at http://algo.inria.fr/seminars/sem01-02/weil.pdf.

[CLPZ02] C. Christopher, J. Llibre, C. Pantazi and X. Zhang. Darboux integrability and invariant algebraic curves for planar polynomial systems. J. Phys. A: Math. Gen. 35 (2002), 2457-2476.

[Con] I. Connell. Elliptic curve packages apecs for maple. Available at $\mathrm{ftp}: / / \mathrm{ftp}$.math.mcgill.ca/pub/apecs.

[Dar78] G. Darboux. Mémoire sur les équations différentielles algébriques du premier ordre et du premier degré. Bull. Sci. Math. (2) 2 (1878), 60-96,123-144,151-200.

[Erd53] A. Erdélyi, (ed). Higher Transcendental Functions, Vol. I. McGraw-Hill Book Company, New York, 1953.

[Fuc75] L. Fuchs. Uber die linearen Differentialgleichungen zweiter Ordnung, welche algebraische Integralen besitzen, und eine neue Anwendung der Invariantentheorie. J. Reine Angew. Math. 81 (1875), 97-147. 
[Ful69] W. Fulton. Algebraic Curves: An introduction to Algebraic Geometry, (Mathematics Lecture Note Series). The Benjamin/Cummings Publishing Company, Inc., Reading, MA, 1969.

[Kat72] N. Katz. Algebraic solutions of differential equations. Invent. Math. 18 (1972), 1-118.

[Kle77] F. Klein. Uber lineare differentialgleichungen I. Math. Ann. 11 (1877), 115-118.

[Kle78] F. Klein. Uber lineare differentialgleichungen II. Math. Ann. 12 (1878), 167-179.

[Kle84] F. Klein. Vorlesungen über das Ikosaeder und die Auflösung del Gleichungen vom fünften Grade. Leipzig, 1884.

[Kov86] J. J. Kovacic. An algorithm for solving second order linear differential equations. J. Symbol. Comput. 2 (1986), 3-43.

[Lit04] R. Litcanu. Lamé operators with finite monodromy - a combinatorial approach. J. Differ. Eqns 207 (2004), 93-116.

[Oll01] J. M. Ollagnier. Liouvillian integration of the Lotka-Volterra system. Qual. Theory Dynam. Syst. 2 (2001), 307-358.

[Pep81] P. Th. Pepin. Méthodes pour obtenir les integrales algebriques des equations differentielles lineaires du second ordre. Atti Accad. Pont. Nouvi Lincei 36 (1881), 243-388.

[Sch72] H. A. Schwarz. Ueber diejenigen Falle, in welchen die Gaussische hypergeometrische Reihe eine algebraische Funktion ihres vierten Elements darstelt. J. Reine Angew. Math. 75 (1872), 292-335.

[Sha74]

[Sil86]

I.R. Shafarevich. Basic Algebraic Geometry (Grundlehren, 213). Springer, Heidelberg, 1974.

[Sin92]

J. H. Silverman. The arithmetic of elliptic curves (Graduate Texts in Mathematics, 106). Springer, New York, 1986. (1992), 673-687.

[SU93] M. F. Singer and F. Ulmer. Liouvillian and algebraic solutions of second and third order linear differential equations. J. Symbol. Comput. 16(3) (1993), 37-73.

[Tak89] N. Takayama. Gröbner basis and the problem of contiguous relations. Japan J. Appl. Math. 6 (1989), $147-160$.

[UW96] F. Ulmer and J.-A. Weil. Note on Kovacic's algorithm. J. Symbol. Comput. 22(2) (1996), 179-200, August 1996.

[vdPU98] M. van der Put and F. Ulmer. Differential equations and finite groups. Technical Report, Math. Sci. Res. Inst., Berkeley, CA, 1998.

[vdW02] H. A. van der Waall. Lamé Equations with Finite Monodromy. PhD Thesis, University of Utrecht, 2002.

[vH94] M. van Hoeij. Computing parametrizations of rational algebraic curves. In ISSAC '94 Proceedings, 1994, pp. 187-196.

[vHW05] M. van Hoeij and J.-A. Weil. Solving second order linear differential equations with Klein's theorem. In Proceedings of the 2005 International Symposium on Symbolic and Algebraic Computation (ISSAC), 2005.

[Vid99] R. Vidūnas. Aspects of Algorithmic Algebra: Differential Equations and Splines. PhD Thesis, University of Groningen, 1999. Available at http://win-www.uia.ac.be/u/vidunas/Phdthesis.ps.gz.

[Vid03] R. Vidūnas. Contiguous relations of hypergeometric series. J. Comput. Appl. Math. 153 (2003), 507-519.

[Vid05] R. Vidunas. Transformations of some Gauss hypergeometric functions. J. Comput. Appl. Math. 178 (2005), 473-487.

[Vid09] R. Vidūnas. Algebraic transformations of Gauss hypergeometric functions. Funkcialaj Ekvacioj 52(2) (2009), 139-180.

[Vid11] R. Vidunas. Dihedral Gauss hypergeometric functions. Kyushu J. Math. 65(1) (2011), 141-167.

[Wei94] J.-A. Weil. The use of special semi-groups for solving quasi-linear differential equations. Proc. ISAAC'94. ACM Press, New York, 1994.

[Wei95] J.-A. Weil. Constantes et polynomes de Darboux en algebre differentelle. PhD Thesis, Ecole Polytechnique, 1995.

\author{
Raimundas Vidūnas \\ Department of Informatics \& Telecommunications \\ National Kapodistrian University of Athens \\ Panepistimiopolis 15784 \\ Greece \\ (E-mail: rvidunas@di.uoa.gr)
}

